\title{
A two-dimensional metastable flame-front and a degenerate spike-layer problem
}

\author{
Alexei F. CheviaKov ${ }^{\dagger}$ AND Michael J. Ward ${ }^{\dagger}$ \\ Department of Mathematics, University of British Columbia, Vancouver, Canada V6T $1 Z 2$
}

[Received 17 November 2006 and in revised form 25 June 2007]

\begin{abstract}
A formal asymptotic analysis is used to analyze the metastable behavior associated with a nonlocal PDE model describing the upward propagation of a flame-front interface in a vertical channel with a two-dimensional convex cross-section. In a certain asymptotic limit, the flame-front interface assumes a roughly paraboloidal shape with the tip of the paraboloid drifting asymptotically exponentially slowly towards the closest point on the wall of the channel. Asymptotic estimates for the exponentially small eigenvalues responsible for this metastable behavior are derived together with an explicit ODE for the slow motion of the tip of the paraboloid. The subsequent slow motion of the tip along the channel wall is also characterized explicitly. The analysis is based on a nonlinear transformation that has the effect of transforming the paraboloidal interface to a spike-layer solution of a specific singularly perturbed quasilinear parabolic problem with a nondifferentiable quasilinear term.
\end{abstract}

\section{Introduction}

We analyze the nonlinear evolution equation of [10] and [15] that models a flame-front propagating upwards in a vertical channel. In [15] and Appendix B of [3] a nonlocal PDE for the flame-front interface was derived by taking into account the competing effects of buoyancy and gravity. In nondimensional variables, and in a certain asymptotic limit, the flame-front interface $S=S(\mathbf{x}, t)$ was found to satisfy

$$
\begin{gathered}
S_{t}-\frac{1}{2}|\nabla S|^{2}=\varepsilon^{2} \Delta S+S-\langle S\rangle, \quad \mathbf{x} \in \Omega, t>0, \\
\partial_{n} S=0, \quad \mathbf{x} \in \partial \Omega ; \quad S(\mathbf{x}, 0)=S_{0}(\mathbf{x}) ; \quad\langle S\rangle \equiv \frac{1}{|\Omega|} \int_{\Omega} S(\mathbf{x}, t) \mathrm{d} \mathbf{x} .
\end{gathered}
$$

Here $\Omega \subset \mathbb{R}^{2}$ is the bounded channel cross-section, $|\Omega|$ is the area of $\Omega$, and $\partial_{n}$ is the outward normal derivative. We assume that $\Omega$ is convex with a smooth boundary $\partial \Omega$. The small parameter $\varepsilon>0$ is defined in terms of the channel width, the gravitational acceleration, and some physical properties of the flame (see equation (1.4) of [3]).

In the one-dimensional case where $\Omega=\{x|| x \mid<1\}$, the numerical results of [10] suggested that the flame-front interface assumes a roughly concave parabolic shape where the tip of the parabola drifts slowly towards one of the endpoints of the interval at $x= \pm 1$. For $\varepsilon \ll 1$, it was proved in [2] and [3] that the speed of this slow drift of the tip of the parabola is asymptotically

\footnotetext{
${ }^{\dagger}$ E-mail: alexch@math.ubc.ca

${ }^{\ddagger}$ E-mail: ward@math.ubc.ca
} 
exponentially small as $\varepsilon \rightarrow 0$. For $\varepsilon \rightarrow 0$, a formal asymptotic analysis was used in [16] to derive the following nonlinear ODE for the tip $x_{0}(t)$ of the flame-front interface for 1.1 :

$$
x_{0}^{\prime} \sim \sqrt{\frac{2}{\pi \varepsilon^{2}}}\left(\left[\left(1-x_{0}\right)^{2}+O\left(\varepsilon^{2}\right)\right] e^{-\left(1-x_{0}\right)^{2} /\left(2 \varepsilon^{2}\right)}-\left[\left(1+x_{0}\right)^{2}+O\left(\varepsilon^{2}\right)\right] e^{-\left(1+x_{0}\right)^{2} /\left(2 \varepsilon^{2}\right)}\right) .
$$

The analyses of [2], [3], and [16] were based on introducing the transformation $y=-S_{x}$ into (1.1) to eliminate the nonlocal term. The resulting PDE problem for $y=y(x, t)$ on $|x|<1$ is the Burgers-Sivashinsky equation

$$
y_{t}+y y_{x}-y=\varepsilon^{2} y_{x x}, \quad y( \pm 1, t)=0, \quad y(x, 0)=-S_{x}(x, 0) .
$$

In a vertical channel with a two-dimensional cross-section, the upwardly propagating flamefront assumes a roughly paraboloidal shape with the tip of the paraboloid located somewhere in the channel cross-section. The numerical results of [1] and [8] strongly indicate that the paraboloidal flame-front interface maintains its shape for a very long time with the tip of the paraboloid drifting asymptotically slowly in $\varepsilon$ towards the boundary of the channel cross-section. Experimental evidence of such long-lived transients from physical experiments with premixed flames are summarized in $\S 2$ of [8]. For the special case of a unit square channel cross-section, it was proved in [1] that the flame-front is metastable in the sense that the tip of the flame-front remains inside $\Omega$ for an asymptotically exponentially long time when $\varepsilon \ll 1$. The method of proof in [1] was based on differentiating (1.1a) separately with respect to $x$ and $y$; then by using comparison principles the resulting problems were related to the Burgers-Sivashinsky equation (1.3) where metastability was proved in [3]. Although this approach proved the existence of a metastable flamefront in a square domain, it left open the issue of providing an explicit analytical characterization of the metastable flame-front dynamics and of providing asymptotic estimates for the exponentially small eigenvalues associated with the linearization around the flame-front. The numerical results in [1] did suggest that the flame-front tip eventually approaches the closest point on the boundary of the square. In addition, Theorem 2 of [1] proves that, under some assumptions on the initial data, the flame-front tip will approach one of the corners of the square. For an elliptical domain, the numerical results of $[8]$ showed that the flame-front tip approaches the closest point on the boundary of the ellipse. From the numerical study of [8], the tip then drifts along the boundary of the ellipse towards the nearest local maximum of the boundary curvature.

The goal of this paper is to extend the results in [1] and [8] by giving an explicit, but formal, asymptotic characterization of metastability for 1.1 when $\Omega$ is a convex domain. We also study the motion of the flame-front tip on the boundary of the domain. Although we provide an explicit asymptotic characterization of the flame-front dynamics for a well-developed paraboloidal flamefront, we do not study the important problem of the formation of the flame-front from arbitrary initial data. In contrast to the rigorous approach in [1] in which (1.1a) is differentiated with respect to $x$ and $y$ to eliminate the nonlocal term, our study of (1.1) is based on a nonlinear change of variables that reduces (1.1) to a quasilinear parabolic problem. In our formulation the paraboloidal flame-front interface with tip at $\mathbf{x}_{0}$ inside $\Omega$ is transformed for $\varepsilon \rightarrow 0$ to a localized spike-layer solution with the spike centered at $\mathbf{x}_{0}$. The metastable behavior of this interior spike-layer solution is then studied in detail using a formal asymptotic analysis. For the simpler one-dimensional case, this approach has recently been used in [14] as an alternative to the metastability analysis of [16] based on the Burgers-Sivashinsky equation (1.3). 

by

Before outlining our specific results, we introduce the transformation of (1.1). We first define $\mathcal{U}$

$$
S(\mathbf{x}, t)=2 \varepsilon^{2} \log [\mathcal{U}(\mathbf{x}, t)] .
$$

Upon substituting (1.4) into $(1.1]$, we find that $\mathcal{U}$ satisfies

$$
\mathcal{U}_{t}=\varepsilon^{2} \Delta \mathcal{U}+\mathcal{U}(\log \mathcal{U}-\langle\log \mathcal{U}\rangle), \quad \mathbf{x} \in \Omega, t>0 ; \quad \partial_{n} \mathcal{U}=0, \quad \mathbf{x} \in \partial \Omega .
$$

Since there is no nontrivial equilibrium solution to 1.5 , we introduce the further change of variables

$$
\mathcal{U}(\mathbf{x}, t)=e^{c(t)+\omega} u(\mathbf{x}, t) .
$$

Here $c(t)$ is chosen so that the problem for $u$ has a steady-state solution, and $\omega$ is an arbitrary constant. Therefore,

$$
c^{\prime}(t)=-\langle\log u(\mathbf{x}, t)\rangle
$$

while $u$ satisfies the quasilinear parabolic problem

$$
u_{t}=\varepsilon^{2} \Delta u+u \log u, \quad x \in \Omega, t>0 ; \quad \partial_{n} u=0, \quad x \in \partial \Omega .
$$

The equilibrium problem for 1.8 in $\mathbb{R}^{2}$ is

$$
\varepsilon^{2} \Delta u^{\infty}+u^{\infty} \log u^{\infty}=0, \quad \mathbf{x} \in \mathbb{R}^{2} ;\left.\quad u^{\infty}\right|_{|\mathbf{x}| \rightarrow \infty}=0 .
$$

As a result of the nondifferentiability of $u \log u$ at $u=0$, this problem is a special case of a class of degenerate spike-layer problems studied in [5]. A remarkable feature of [1.9] is that it has the simple exact spike-layer solution

$$
u^{\infty}\left(\mathbf{x} ; \mathbf{x}_{0}\right)=\exp \left(1-\frac{\left|\mathbf{x}-\mathbf{x}_{0}\right|^{2}}{4 \varepsilon^{2}}\right), \quad \mathbf{x} \in \mathbb{R}^{2},
$$

for any fixed spike location $\mathbf{x}_{0} \in \mathbb{R}^{2}$. This is the unique solution with $u^{\infty}>0$ to $[1.9$ ) (cf. [5]).

Since the spike profile $u^{\infty}$ only fails to satisfy the boundary conditions of the finite-domain problem 1.8 by asymptotically exponentially small terms as $\varepsilon \rightarrow 0$ for any $\mathbf{x}_{0} \in \Omega, u^{\infty}$ is an approximate steady-state solution of (1.8). Upon substituting $u=u^{\infty},(1.7)$, and $(1.6)$, into (1.4), we obtain

$$
S(\mathbf{x}, t)=\frac{1}{2} \int_{0}^{t}\left\langle\left|\mathbf{x}-\mathbf{x}_{0}\right|^{2}\right\rangle \mathrm{d} \tau-\frac{1}{2}\left|\mathbf{x}-\mathbf{x}_{0}\right|^{2}+2 \varepsilon^{2}(1-t+\omega) .
$$

Therefore, the spike profile $u^{\infty}$, with spike location at $\mathbf{x}_{0}$ is transformed to a paraboloidal flamefront interface, with tip at $\mathbf{x}_{0}$, that propagates upwards in the channel at speed $v(t)=2 \varepsilon^{2} c^{\prime}=$ $\frac{1}{2}\left\langle\left|\mathbf{x}-\mathbf{x}_{0}\right|^{2}\right\rangle+O\left(\varepsilon^{2}\right)$. In Fig. 1 we show a plot of the interface $w=\log u^{\infty}=S /\left(2 \varepsilon^{2}\right)$ and the corresponding spike profile $u^{\infty}$ from $(1.10)$ for the domain $\Omega=[-1,1] \times[-1,1]$ when $\mathbf{x}_{0}(0)=(0.1,0.3)$ and $\varepsilon=0.1$. However, since the form for $S$ in (1.11) does not satisfy $\partial_{n} S=0$ on $\partial \Omega$, (1.11) must be modified near $\partial \Omega$ by introducing certain boundary-layer terms. For the time-dependent problem, we allow $\mathbf{x}_{0}$ and $\omega$ to depend slowly on time. By using formal asymptotic methods to resolve the boundary layer near $\partial \Omega$, we will derive slow motion ODE's for $\mathbf{x}_{0}(t)$ and for $\omega(t)$. By substituting these slow motion ODE's into $(1.11)$, we obtain an explicit characterization of the metastability for the flame-front interface of $(1.1)$. The precise metastability result is given in 


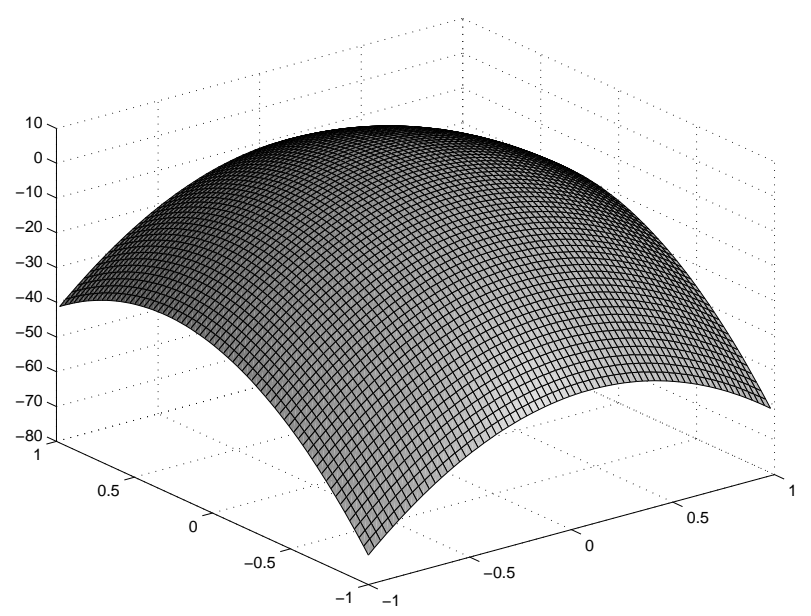

(a) interface

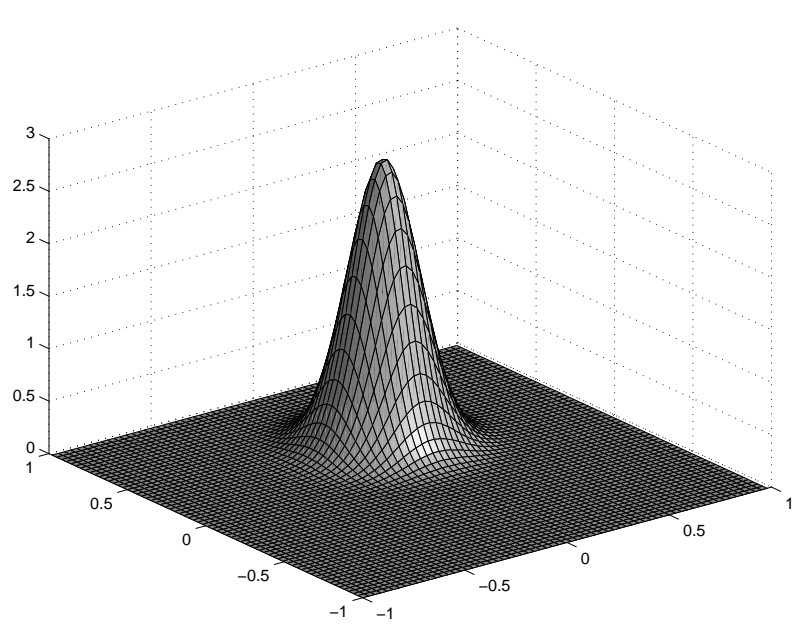

(b) spike profile

FIG. 1. Plot of the interface $w=S /\left(2 \varepsilon^{2}\right) \equiv 1-\left|\mathbf{x}-\mathbf{x}_{0}\right|^{2} /\left(4 \varepsilon^{2}\right)$ (top), and the spike profile $u^{\infty}$ from 1.10 (bottom), when $\mathbf{x}_{0}=(0.1,0.3)$ and $\varepsilon=0.1$. The domain is the square $\Omega=[-1,1] \times[-1,1]$.

Principal Result 4.1 of $\S 4$ below. For $\varepsilon \rightarrow 0$, it is shown that the flame-front tip drifts asymptotically exponentially slowly towards the closest point on the wall of the channel. Illustrations of this phenomena are given in $\S 4$. We also analyze the motion of the flame-front tip after it has become attached to the wall of the channel. By deriving an ODE for the flame-front tip, it is shown that the tip slides along the boundary until it reaches a local maximum of the curvature of the boundary. The 


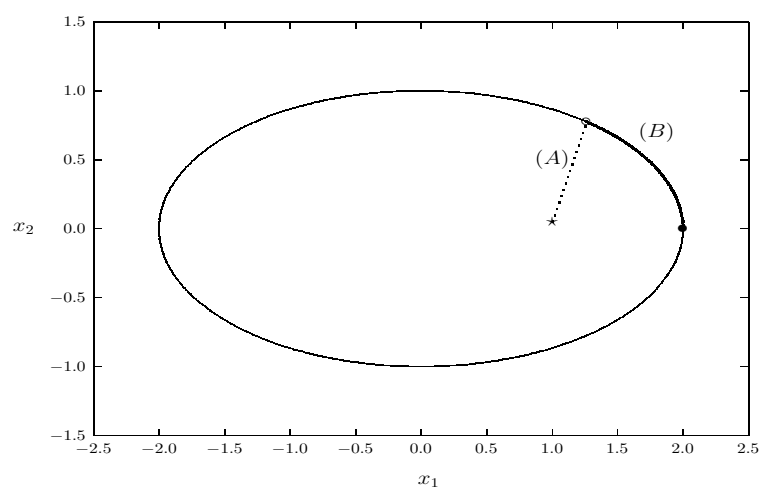

(a) elliptical domain

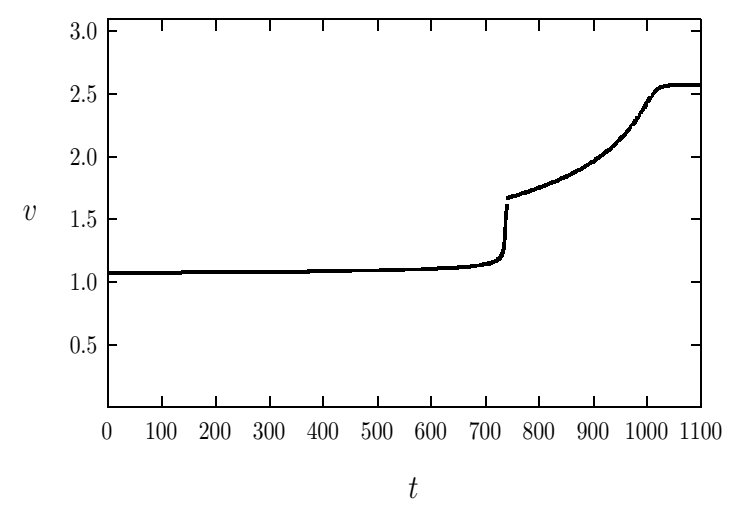

(b) $v$ versus $t$

FIG. 2. Top: Dynamics of the flame-front tip in an ellipse. (A) Under the dynamics 4.17 the tip moves from the initial point (labeled by $\star$ ) to an $O(\epsilon)$ neighborhood of the closest boundary point (labeled by $\circ$ ). (B) The tip then drifts along the boundary under 5.13 to the nearest local maximum of the curvature (labeled by $\bullet$ ). Bottom: the upward speed $v(t)$ of the flame-front given in 6.3.

precise result for the boundary flame-front tip motion is given in Principal Result 5.1 of $\S 5$. These asymptotic results give a formal confirmation of the numerical results and conjectures in [1] and [8]. Similar to Fig. 4 of [8], in Fig. 2(a) we illustrate the two distinct stages of the dynamics of a flamefront tip that is initially located inside an elliptical domain. In Fig. 2(b) we plot the upward speed $v(t)$ of the flame-front in the channel during both stages of the dynamics. An explicit description of the flame-front dynamics for these two stages together with the parameter values used for (1.1) is given in Example 6.1 of $\S 6$.

Equilibrium spike-layer problems similar to $(1.8)$, but with $u \log u$ replaced by either $-u+u^{p}$ or a more general class of differentiable functions of $u$, have been studied extensively over the 
past ten years. Early studies of interior equilibrium spikes include [23], [21], and [18]. For early studies of boundary spikes see [13] and [22]. Metastable spike behavior in two spatial dimensions associated with the nonlocal quasilinear shadow Gierer-Meinhardt problem is analyzed in [6], [21], and [4]. A formal asymptotic analysis of the dynamics of boundary spikes for this shadow GiererMeinhardt model is given in [7]. A recent survey of rigorous properties of equilibrium spike-layer behavior is given in Section 1 of [12]. A survey of metastable behavior and boundary dynamics of spike and bubble solutions for scalar quasilinear problems in two spatial dimensions is given in [19]. A more general survey of formal asymptotic methods for equilibrium and time-dependent spike-layer solutions is given in [20]. In contrast to these previous studies, the study of spike-layer behavior for $(1.8)$ is technically somewhat more challenging owing to the nondifferentiability of the quasilinear term $u \log u$ at $u=0$.

The outline of this paper is as follows. For $\varepsilon \rightarrow 0$, in $\S 2$ we study the spectral problem associated with linearizing (1.8) around the spike profile $u^{\infty}$. By using boundary-layer theory to calculate the boundary behavior of certain near-translation eigenfunctions, precise asymptotic estimates for the asymptotically exponentially small eigenvalues associated with the linearization are derived. In addition, we derive an asymptotic estimate for the $O(1)$ positive principal eigenvalue of the linearization. In $\S 3$ we construct an improved approximation to the equilibrium solution of (1.8) whereby the spike profile $u^{\infty}$ is adjusted by a boundary-layer solution of exponentially small amplitude in order to satisfy $\partial_{n} u=0$ on $\partial \Omega$. We then study the spectral properties of the linearization of (1.8) around this improved quasi-equilibrium solution, and we derive asymptotic estimates for the exponentially small eigenvalues associated with the near-translation invariance. In $\S 4$ we use the spectral estimates of $\$ 2$ to derive explicit slow motion ODE's for the flame-front tip $\mathbf{x}_{0}(t)$ and the growth $\omega(t)$. In $\S 5$ we analyze the dynamics of the flame-front on the boundary of the domain. Finally, in $\S 6$ we conclude with a brief discussion and we illustrate our asymptotic results with a few examples.

\section{The eigenvalue problem: leading order theory}

In this section we analyze the spectral problem associated with linearizing (1.8) around the spike profile $u^{\infty}$ of $(1.10)$. We assume that the spike location $\mathbf{x}_{0} \in \Omega$ satisfies $\operatorname{dist}\left(\mathbf{x}_{0}, \partial \Omega\right)=O(1)$ as $\varepsilon \rightarrow 0$. If we linearize (1.8) around $u^{\infty}$, and neglect the no-flux boundary condition, we obtain the infinite-domain eigenvalue problem

$$
\varepsilon^{2} \Delta \phi^{\infty}+\left(2-\frac{\left|\mathbf{x}-\mathbf{x}_{0}\right|^{2}}{4 \varepsilon^{2}}\right) \phi^{\infty}=\lambda^{\infty} \phi^{\infty}, \quad \mathbf{x} \in \mathbb{R}^{2} ; \quad \phi^{\infty} \rightarrow 0 \quad \text { as }|\mathbf{x}| \rightarrow \infty .
$$

The first three eigenpairs of 2.1], ranked according to the largest eigenvalues, are

$$
\begin{array}{ll}
\lambda_{0}^{\infty}=1, & \phi_{0}^{\infty}=u^{\infty}=\exp \left(1-\frac{\left|\mathbf{x}-\mathbf{x}_{0}\right|^{2}}{4 \varepsilon^{2}}\right), \\
\lambda_{i}^{\infty}=0, & \phi_{i}^{\infty}=\partial_{i} u^{\infty}, \quad i=1,2 .
\end{array}
$$

The result in 2.2b expresses the translation invariance of the infinite-domain problem, and is readily seen by differentiating (1.9) with respect to $x_{i}$ for $i=1,2$. Moreover, since (2.1) is the explicitly solvable 2-D harmonic oscillator eigenvalue problem (cf. [11]), its entire spectrum is

$$
\phi^{\infty}=c_{n_{1}, n_{2}} e^{-|\mathbf{y}|^{2} / 4} \mathrm{He}_{n_{1}}\left(y_{1}\right) \mathrm{He}_{n_{2}}\left(y_{2}\right), \quad \lambda^{\infty}=1-n_{1}-n_{2}, \quad n_{1}, n_{2}=0,1,2, \ldots
$$


Here $\mathbf{y} \equiv \varepsilon^{-1}\left(\mathbf{x}-\mathbf{x}_{0}\right), y_{i}$ is the $i^{\text {th }}$ coordinate of $\mathbf{y}$, and $\operatorname{He}_{n}\left(y_{i}\right)$ is the usual Hermite polynomial of degree $n$. The first three eigenpairs in (2.3) agree with those in (2.2), and the remaining eigenvalues are strictly negative.

If we insist that $\phi$ satisfy the no-flux condition on $\partial \Omega$, then we obtain the finite-domain eigenvalue problem

$$
\mathcal{L}_{0} \phi \equiv \varepsilon^{2} \Delta \phi+\left(1+\log u^{\infty}\right) \phi=\lambda \phi, \quad \mathbf{x} \in \Omega ; \quad \partial_{n} \phi=0, \quad \mathbf{x} \in \partial \Omega .
$$

Since $u^{\infty}$ decays exponentially away from $\mathbf{x}_{0}$, the spectrum of 2.1 should be exponentially close to that of 2.4. In particular, we show formally that the two zero eigenvalues in $2.2 \mathrm{~b}$ associated with translation invariance of the infinite-domain problem become asymptotically exponentially small as $\varepsilon \rightarrow 0$. These eigenvalues are referred to as the critical spectrum. In addition, we will show that the principal eigenvalue of (2.4) is asymptotically exponentially close to the value $\lambda_{0}^{\infty}=1$ given in $2.2 \mathrm{a}$. The eigenfunctions in 2.2 for the infinite-domain problem 2.1 provide outer approximations, valid away from $\partial \Omega$, for corresponding eigenfunctions of the finite-domain problem (2.4). The formulae derived in this section are needed below in $\S 4$ for the metastability analysis.

We begin by writing (2.4) in terms of normal-tangential coordinates $(\sigma, s)$, where $\sigma>0$ is the normal distance from $\partial \Omega$ to $\mathbf{x} \in \Omega$, and $s$ is arclength along $\partial \Omega$. Let $\mathbf{x}(s)=\left(x_{1}(s), x_{2}(s)\right)$ smoothly parameterize $\partial \Omega$ in the positive direction. Then $\mathbf{e}_{s}=\mathbf{x}^{\prime}(s)=\left(x_{1}^{\prime}(s), x_{2}^{\prime}(s)\right)$ is a unit vector tangent to $\partial \Omega$ in the positive direction, and $\mathbf{e}_{\sigma}=\left(-x_{2}^{\prime}(s), x_{1}^{\prime}(s)\right)$ is a unit vector normal to $\partial \Omega$ pointing into $\Omega$. The curvature $\kappa(s) \geqslant 0$ of $\partial \Omega$ is

$$
\kappa(s)=x_{1}^{\prime}(s) x_{2}^{\prime \prime}(s)-x_{2}^{\prime}(s) x_{1}^{\prime \prime}(s)=\mathbf{e}_{\sigma} \cdot \mathbf{e}_{s}^{\prime}=-\mathbf{e}_{\sigma}^{\prime} \cdot \mathbf{e}_{s},
$$

so that $\partial_{s} \mathbf{e}_{\sigma}=-\kappa \mathbf{e}_{s}$ and $\partial_{s} \mathbf{e}_{s}=\kappa \mathbf{e}_{\sigma}$. We then define $\mathbf{d}(s), D(s)$, and $\chi(s)$ by

$$
\mathbf{d}=\mathbf{x}_{0}-\mathbf{x}(s), \quad D=|\mathbf{d}|^{2}, \quad \chi=\frac{1}{2}\left(\mathbf{d}(s) \cdot \mathbf{e}_{\sigma}\right) .
$$

This normal-tangential coordinate system is shown in Fig. 3 In terms of $(\sigma, s)$ we readily calculate that

$$
\begin{aligned}
& \cos \beta=\frac{\mathbf{d} \cdot \mathbf{e}_{\sigma}}{|\mathbf{d}|}, \\
& \left|\mathbf{x}-\mathbf{x}_{0}\right|^{2}=|\mathbf{d}|^{2}+\sigma^{2}-2 \sigma|\mathbf{d}| \cos \beta=|\mathbf{d}|^{2}+\sigma^{2}-2 \sigma\left(\mathbf{d} \cdot \mathbf{e}_{\sigma}\right)=D+\sigma^{2}-4 \chi \sigma .
\end{aligned}
$$

Therefore, in terms of the coordinates $(\sigma, s)$, the spike-profile outer solution 1.10$)$ becomes

$$
u^{\infty}=\exp \left(1-\frac{1}{4 \varepsilon^{2}}\left(D+\sigma^{2}-4 \chi \sigma\right)\right) .
$$

In addition, in terms of $(\sigma, s)$ the Laplacian in 2.4$)$ can be written as

$$
\Delta \phi=\phi_{\sigma \sigma}-\frac{\kappa}{1-\kappa \sigma} \phi_{\sigma}+\frac{1}{1-\kappa \sigma} \frac{\partial}{\partial s}\left(\frac{1}{1-\kappa \sigma} \phi_{s}\right) .
$$

We now construct the two near-translation eigenfunctions of 2.4 . Let $\phi^{\infty}$ denote one of the two translation eigenfunctions for the infinite-domain problem (2.1) normalized as

$$
\phi^{\infty}=\varepsilon^{2} \partial_{i} u^{\infty}, \quad i=1,2 .
$$




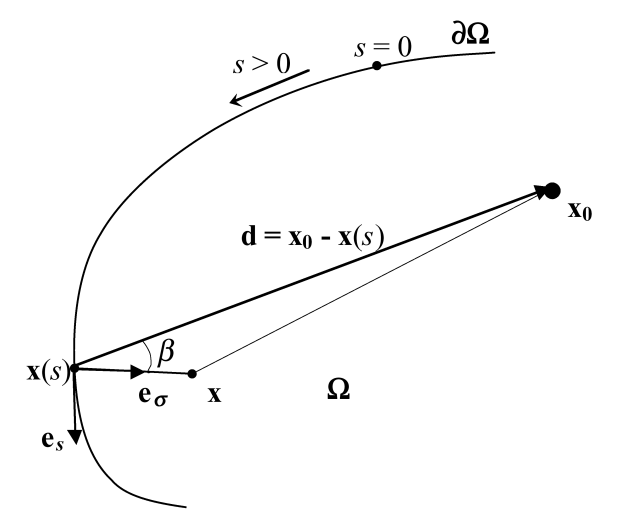

FIG. 3. Coordinate system for the boundary layer analysis.

Here $\partial_{i}$ denotes the partial derivative with respect to the $i^{\text {th }}$ coordinate $x_{i}$ of $\mathbf{x}$. The factor $\varepsilon^{2}$ in 2.10 is chosen so that $\phi^{\infty}=O(1)$ for $\left|\mathbf{x}-\mathbf{x}_{0}\right|=O(\varepsilon)$. Since $\phi^{\infty}$ does not satisfy the no-flux boundary condition on $\partial \Omega$, we construct the two critical eigenfunctions in the additive boundary-layer form

$$
\phi=\phi^{\infty}+\phi_{B}
$$

The correction $\phi_{B}$, which allows the no-flux condition on $\partial \Omega$ to be satisfied, is localized near $\partial \Omega$ and is to decay away from the boundary layer. Such an additive boundary-layer construction is typical in wave scattering problems (cf. [9]). Substituting (2.11) into 2.4), assuming that $\lambda$ is exponentially small, and using 2.8 for $u^{\infty}$, we get

$$
\varepsilon^{2} \Delta \phi_{B}+\left(2-\frac{D}{4 \varepsilon^{2}}+\frac{\chi \sigma}{\varepsilon^{2}}-\frac{\sigma^{2}}{4 \varepsilon^{2}}\right) \phi_{B}=0, \quad \mathbf{x} \in \Omega ; \quad \partial_{\sigma} \phi_{B}=-\partial_{\sigma} \phi^{\infty}, \quad \mathbf{x} \in \partial \Omega .
$$

The problem 2.12) suggests the boundary-layer thickness $O\left(\varepsilon^{2}\right)$ near $\partial \Omega$. Therefore, we introduce the local normal distance $\eta$ by $\eta=\sigma / \varepsilon^{2}$. By differentiating 1.10 , and writing the result in terms of the local boundary-layer coordinates $(\eta, s)$, we obtain

$$
\begin{aligned}
\phi^{\infty} & =\varepsilon^{2} \partial_{i} u^{\infty}=\frac{1}{2}\left(x_{0 i}-x_{i}\right) \exp \left(1-\frac{\left|\mathbf{x}-\mathbf{x}_{0}\right|^{2}}{4 \varepsilon^{2}}\right) \\
& \sim \frac{1}{2} \mathbf{e}_{i} \cdot\left(\mathbf{d}-\varepsilon^{2}\left[\mathbf{e}_{\sigma} \eta+\mathbf{d} \frac{\eta^{2}}{4}\right]\right) \exp \left(1-\frac{D}{4 \varepsilon^{2}}+\eta \chi\right) .
\end{aligned}
$$

Here $\mathbf{e}_{i}$ is the unit vector in the direction of the $i^{\text {th }}$ coordinate, while $\mathbf{d}, \mathbf{e}_{\sigma}, D$, and $\chi$, are as defined in 2.6 and Fig. 3 By differentiating 2.13, and evaluating the result on $\partial \Omega$ where $\sigma=0$, we obtain

$$
\begin{aligned}
\left.\partial_{\sigma} \phi^{\infty}\right|_{\sigma=0} & =\left.\mathbf{e}_{\sigma} \cdot \nabla \phi^{\infty}\right|_{\sigma=0}=\mathbf{e}_{\sigma} \cdot\left(\frac{d_{i}}{4 \varepsilon^{2}} \mathbf{d}-\frac{\mathbf{e}_{i}}{2}\right) \exp \left(1-\frac{D}{4 \varepsilon^{2}}\right) \\
& =\frac{1}{2 \varepsilon^{2}}\left(d_{i} \chi-\varepsilon^{2} \mathbf{e}_{i} \cdot \mathbf{e}_{\sigma}\right) \exp \left(1-\frac{D}{4 \varepsilon^{2}}\right)
\end{aligned}
$$


Since $\partial_{\sigma} \phi_{B}=-\partial_{\sigma} \phi^{\infty}$ for $\mathbf{x} \in \partial \Omega, 2.14$ gives the following boundary condition for $\phi_{B}$ :

$$
\left.\partial_{\sigma} \phi_{B}\right|_{\sigma=0}=-\frac{1}{2 \varepsilon^{2}}\left(d_{i} \chi-\varepsilon^{2} \mathbf{e}_{i} \cdot \mathbf{e}_{\sigma}\right) \exp \left(1-\frac{D}{4 \varepsilon^{2}}\right) .
$$

Next, we seek a boundary-layer solution to (2.12) with boundary condition 2.15) in the form

$$
\phi_{B}=\exp \left(1-\frac{D}{4 \varepsilon^{2}}\right) \Phi_{B}, \quad \Phi_{B}=\Phi_{B 0}+\varepsilon^{2} \Phi_{B 1}+\cdots, \quad \eta=\sigma / \varepsilon^{2},
$$

where $D(s)=\left|\mathbf{x}(s)-\mathbf{x}_{0}\right|^{2}$ and $\mathbf{x}(s) \in \partial \Omega$. We then substitute 2.16 into 2.12 and 2.15) after first expressing the Laplacian in 2.12) in terms of normal-tangential coordinates as in (2.9). Upon collecting the lowest powers of $\varepsilon$, we find that $\Phi_{B 0}$ satisfies

$$
\begin{aligned}
& \left(\Phi_{B 0}\right)_{\eta \eta}-\frac{1}{16}\left[4 D-\left(D^{\prime}\right)^{2}\right] \Phi_{B 0}=0, \quad 0 \leqslant \eta<\infty ; \\
& \left.\left(\Phi_{B 0}\right)_{\eta}\right|_{\eta=0}=-d_{i} \chi / 2,\left.\quad \Phi_{B 0}\right|_{\eta \rightarrow \infty} \rightarrow 0 .
\end{aligned}
$$

The coefficient of $\Phi_{B 0}$ in 2.17) can be simplified by noting that $D^{\prime}=\left(|\mathbf{d}|^{2}\right)^{\prime}=2 \mathbf{d} \cdot \mathbf{d}^{\prime}=-2 \mathbf{d} \cdot \mathbf{e}_{s}$. In addition, since $\left|\mathbf{e}_{s}\right|=\left|\mathbf{e}_{\sigma}\right|=1$, we obtain $D=|\mathbf{d}|^{2}=\left(\mathbf{d} \cdot \mathbf{e}_{s}\right)^{2}+\left(\mathbf{d} \cdot \mathbf{e}_{\sigma}\right)^{2}$. This yields

$$
\frac{D}{4}-\frac{\left(D^{\prime}\right)^{2}}{16}=\frac{1}{4}\left(\mathbf{d} \cdot \mathbf{e}_{\sigma}\right)^{2}=\chi^{2}
$$

Therefore, the solution to 2.17) is

$$
\Phi_{B 0}=\frac{d_{i}}{2} e^{-\chi \eta}
$$

For a convex domain, $\chi=\mathbf{d} \cdot \mathbf{e}_{\sigma} / 2>0$, and so $\Phi_{B 0}$ decays exponentially away from the boundary layer as $\eta \rightarrow \infty$.

Finally, by substituting 2.19, 2.16, and 2.13 into 2.11, and evaluating the resulting expression on $\partial \Omega$, we obtain the following boundary behavior for the critical eigenfunctions of 2.4):

$$
\left.\left.\phi\right|_{\eta=0} \sim \phi^{\infty}\right|_{\eta=0}+\left.\exp \left(1-\frac{D}{4 \varepsilon^{2}}\right) \Phi_{B 0}\right|_{\eta=0} \sim d_{i} \exp \left(1-\frac{D}{4 \varepsilon^{2}}\right) .
$$

Next, we use 2.20 to give an estimate of the two exponentially small eigenvalues for 2.4 corresponding to the near-translation eigenfunctions. By using Green's identity on 2.4 we get

$$
\int_{\Omega}\left[\partial_{i} u^{\infty} \mathcal{L}_{0} \phi-\phi \mathcal{L}_{0}\left(\partial_{i} u^{\infty}\right)\right] \mathrm{d} \mathbf{x}=\int_{\partial \Omega} \varepsilon^{2}\left[\partial_{i} u^{\infty} \partial_{n} \phi-\phi \partial_{n}\left(\partial_{i} u^{\infty}\right)\right] \mathrm{d} s .
$$

Then, since $\mathcal{L}_{0}\left(\partial_{i} u^{\infty}\right)=0, \mathcal{L}_{0} \phi=\lambda \phi$, and $\partial_{n} \phi=0$ on $\partial \Omega$, 2.21 becomes

$$
\lambda \int_{\Omega} \phi \partial_{i} u^{\infty} \mathrm{d} \mathbf{x}=-\varepsilon^{2} \int_{\partial \Omega} \phi \partial_{n}\left[\partial_{i} u^{\infty}\right] \mathrm{d} s .
$$

In (2.22), $\partial_{n}$ denotes the outward normal derivative. The dominant contribution to the integral multiplying $\lambda$ occurs from the region near the spike where $\phi \sim \varepsilon^{2} \partial_{i} u^{\infty}$ from 2.10). Therefore, (2.22) becomes

$$
\lambda \mathcal{J} \sim-\mathcal{I} ; \quad \mathcal{J} \equiv \varepsilon^{2} \int_{\Omega}\left(\partial_{i} u^{\infty}\right)^{2} \mathrm{~d} \mathbf{x}, \quad \mathcal{I} \equiv \varepsilon^{2} \int_{\partial \Omega} \phi \partial_{n}\left[\partial_{i} u^{\infty}\right] \mathrm{d} s .
$$


We will estimate $\mathcal{J}$ and $\mathcal{I}$ precisely as $\varepsilon \rightarrow 0$. To calculate $\mathcal{J}$ in 2.23 we use 1.10 for $u^{\infty}$ to obtain

$$
\mathcal{J} \sim \frac{e^{2}}{4 \varepsilon^{2}} \int_{\Omega}\left(x_{i}-x_{0 i}\right)^{2} e^{-\left|\mathbf{x}-\mathbf{x}_{0}\right|^{2} /\left(2 \varepsilon^{2}\right)} \mathrm{d} \mathbf{x} \sim \frac{\pi e^{2} \varepsilon^{2}}{4} \int_{0}^{\infty} \rho^{3} e^{-\rho^{2} / 2} \mathrm{~d} \rho=\frac{\pi e^{2} \varepsilon^{2}}{2} .
$$

Next, we calculate $\mathcal{I}$. To do so, we evaluate $\partial_{i} u^{\infty}$ as in 2.13 and calculate its outward normal derivative as

$$
\left.\partial_{n}\left[\partial_{i} u^{\infty}\right]\right|_{\eta=0}=-\left.\varepsilon^{-2} \partial_{\eta}\left[\partial_{i} u^{\infty}\right]\right|_{\eta=0} \sim-\frac{\chi d_{i}}{2 \varepsilon^{4}} \exp \left(1-\frac{D}{4 \varepsilon^{2}}\right) .
$$

Upon substituting (2.25) and (2.20) into 2.23 we obtain $\mathcal{I}$. Then, substituting the resulting expression and (2.24) into the expression for $\lambda$ in (2.23), we obtain the following result for the critical spectrum:

PRINCIPAL RESUlt 2.1 For $\varepsilon \rightarrow 0$, the two exponentially small eigenvalues of the finite-domain eigenvalue problem 2.4 corresponding to the near-translation eigenfunctions have the asymptotic estimate

$$
\lambda \sim \frac{1}{\pi \varepsilon^{4}} \int_{\partial \Omega} d_{i}^{2} \chi e^{-D /\left(2 \varepsilon^{2}\right)} \mathrm{d} s .
$$

Here $2 \chi=\mathbf{d} \cdot \mathbf{e}_{\sigma}, D=\left|\mathbf{x}(s)-\mathbf{x}_{0}\right|^{2}$, and $d_{i}=x_{0 i}-x_{i}(s)$.

Next, we use Laplace's method (cf. [24]) to asymptotically evaluate the integral in 2.26 for $\varepsilon \ll 1$. For $\varepsilon \rightarrow 0$, the dominant contribution to this integral arises from the point $s_{0}$ on $\partial \Omega$ closest to the spike location $\mathbf{x}_{0}$. Assume that there is only one such point where $D(s)$ takes its global minimum for $s \in \partial \Omega$. At $s=s_{0}$ we get $\chi^{2}=D / 4$ from 2.18$)$ and $D^{\prime \prime}\left(s_{0}\right)=2\left(1-\left|\mathbf{d}_{0}\right| \kappa\right)$. Therefore, Laplace's method applied to 2.26 gives the following leading-order asymptotic estimate for the two exponentially small eigenvalues of 2.4):

$$
\lambda \sim \frac{\varepsilon^{-3}}{\sqrt{2 \pi}} \frac{d_{i 0}^{2}\left|\mathbf{d}_{0}\right|}{\sqrt{1-\kappa_{0}\left|\mathbf{d}_{0}\right|}} \exp \left(-\frac{\left|\mathbf{d}_{0}\right|^{2}}{2 \varepsilon^{2}}\right)
$$

Here $\left|\mathbf{d}_{0}\right| \equiv\left|\mathbf{x}_{0}-\mathbf{x}\left(s_{0}\right)\right|, d_{i 0}$ is the $i^{\text {th }}$ coordinate of $\mathbf{d}$ at $s=s_{0}$, and $\kappa_{0} \equiv \kappa\left(s_{0}\right)$ is the curvature of $\partial \Omega$ at $s=s_{0}$.

Finally, we derive an estimate for the principal eigenvalue of 2.4. This eigenvalue is exponentially close to one, and the outer approximation for its corresponding eigenfunction, valid away from $\partial \Omega$, is $\phi \sim u^{\infty}$. To derive an estimate for $\lambda-1$ we use Green's identity on 2.4 to get

$$
\int_{\Omega}\left[u^{\infty} \mathcal{L}_{0} \phi-\phi \mathcal{L}_{0} u^{\infty}\right] \mathrm{d} \mathbf{x}=\int_{\partial \Omega} \varepsilon^{2}\left[u^{\infty} \partial_{n} \phi-\phi \partial_{n} u^{\infty}\right] \mathrm{d} s .
$$

From 1.9 we note that $\mathcal{L}_{0} u^{\infty}=u^{\infty}$. In addition, since $\partial_{n} \phi=0$ on $\partial \Omega, 2.28$ reduces to

$$
(\lambda-1) \int_{\Omega} u^{\infty} \phi \mathrm{d} \mathbf{x}=-\varepsilon^{2} \int_{\partial \Omega} \phi \partial_{n} u^{\infty} \mathrm{d} s .
$$

The dominant contribution to the integral multiplying $\lambda-1$ occurs from the region near the spike where $\phi \sim u^{\infty}$. Therefore, from 1.10 we estimate

$$
\int_{\Omega} u^{\infty} \phi \mathrm{d} \mathbf{x} \sim \int_{\Omega}\left(u^{\infty}\right)^{2} \mathrm{~d} \mathbf{x} \sim 2 \pi e^{2} \int_{0}^{\infty} \rho e^{-\rho^{2} /\left(2 \varepsilon^{2}\right)} \mathrm{d} \rho=2 \pi \varepsilon^{2} e^{2} .
$$


In addition, by differentiating (2.8) with respect to $\sigma$, we readily calculate that

$$
\left.\partial_{n} u^{\infty}\right|_{\partial \Omega}=-\left.\partial_{\sigma} u^{\infty}\right|_{\sigma=0}=-\frac{\chi}{\varepsilon^{2}} \exp \left(1-\frac{D}{4 \varepsilon^{2}}\right) .
$$

To complete the evaluation of the right-hand side of 2.29 we must calculate $\phi$ on $\partial \Omega$ by using boundary-layer theory. We begin by writing (2.4) in the form

$$
\varepsilon^{2} \Delta \phi+\left(\log u^{\infty}\right) \phi=(\lambda-1) \phi, \quad \mathbf{x} \in \Omega ; \quad \partial_{n} \phi=0, \quad \mathbf{x} \in \partial \Omega .
$$

We look for a boundary-layer solution in the form $\phi=u^{\infty}+\phi_{B}$, where $\phi_{B}$ decays to zero away from $\partial \Omega$. Assuming that $\lambda-1$ is exponentially small, and by using 2.8 for $u^{\infty}$, we find that $\phi_{B}$ satisfies

$$
\varepsilon^{2} \Delta \phi_{B}+\left(1-\frac{D}{4 \varepsilon^{2}}+\frac{\chi \sigma}{\varepsilon^{2}}-\frac{\sigma^{2}}{4 \varepsilon^{2}}\right) \phi_{B}=0, \quad \mathbf{x} \in \Omega ;\left.\quad \partial_{\sigma} \phi_{B}\right|_{\sigma=0}=-\frac{\chi}{\varepsilon^{2}} \exp \left(1-\frac{D}{4 \varepsilon^{2}}\right) .
$$

Next, we look for a solution to 2.33 in the form 2.16). We then substitute 2.16 into 2.33) and introduce the local normal-tangential coordinates $(\eta, s)$, where $\eta=\sigma / \varepsilon^{2}$. To leading order we find that $\Phi_{B 0}$ in 2.16) satisfies

$$
\left(\Phi_{B 0}\right)_{\eta \eta}-\chi^{2} \Phi_{B 0}=0, \quad 0 \leqslant \eta<\infty ;\left.\quad\left(\Phi_{B 0}\right)_{\eta}\right|_{\eta=0}=-\chi .
$$

The solution to 2.34 is $\Phi_{B 0}=e^{-\chi \eta}$. Then, by using $\phi=u^{\infty}+\phi_{B}$ and 2.8, we obtain the boundary estimate

$$
\left.\phi\right|_{\sigma=0} \sim 2 \exp \left(1-\frac{D}{4 \varepsilon^{2}}\right) .
$$

To obtain the estimate for $\lambda-1$ we substitute 2.30, 2.31, and 2.35) into 2.29). This leads to the following result, which is required in the metastability analysis of $\S 4$ :

PRINCIPAL RESUlt 2.2 For $\varepsilon \rightarrow 0$, the principal eigenvalue of the finite-domain eigenvalue problem [2.4], corresponding to the eigenfunction with outer approximation $\phi \sim u^{\infty}$, has the asymptotic estimate

$$
\lambda-1 \sim \frac{1}{\pi \varepsilon^{2}} \int_{\partial \Omega} \chi e^{-D /\left(2 \varepsilon^{2}\right)} \mathrm{d} s .
$$

Here $2 \chi=\mathbf{d} \cdot \mathbf{e}_{\sigma}$ and $D(s)=\left|\mathbf{x}(s)-\mathbf{x}_{0}\right|^{2}$. Assuming that there is a unique point $s=s_{0}$ on $\partial \Omega$ where $D(s)$ is minimized, and defining $d_{0} \equiv\left|\mathbf{x}_{0}-\mathbf{x}\left(s_{0}\right)\right|$ and $\kappa_{0} \equiv \kappa\left(s_{0}\right)$, Laplace's method applied to 2.36 yields the leading-order estimate

$$
\lambda-1 \sim \frac{1}{\sqrt{2 \pi}} \frac{d_{0} \varepsilon^{-1}}{\sqrt{1-\kappa_{0} d_{0}}} e^{-d_{0}^{2} /\left(2 \varepsilon^{2}\right)} .
$$

\section{The eigenvalue analysis: higher order theory}

In this section we use the method of matched asymptotic expansions to construct a quasi-equilibrium spike-type solution $u_{\varepsilon}$ to the finite-domain problem

$$
\varepsilon^{2} \Delta u+u \log u=0, \quad \mathbf{x} \in \Omega ; \quad \partial_{n} u=0, \quad \mathbf{x} \in \partial \Omega .
$$


The finite-domain eigenvalue problem associated with linearizing (1.8) around $u_{\varepsilon}$ is

$$
\mathcal{L}_{\varepsilon} \phi \equiv \varepsilon^{2} \Delta \phi+\left(1+\log u_{\varepsilon}\right) \phi=\lambda \phi, \quad \mathbf{x} \in \Omega ; \quad \partial_{n} \phi=0, \quad \mathbf{x} \in \partial \Omega .
$$

We will derive precise asymptotic estimates for the two critical eigenfunctions and eigenvalues of (3.2) associated with the near-translation invariance.

We first construct $u_{\varepsilon}$ using boundary-layer theory. The outer solution for 3.1, valid away from $\partial \Omega$, is the spike profile $u^{\infty}$ of $(1.10)$, where $\mathbf{x}_{0} \in \Omega$ and $\operatorname{dist}\left(x_{0}, \partial \Omega\right)=O(1)$ as $\varepsilon \rightarrow 0$. Since $u^{\infty}$ fails to satisfy the boundary condition in 3.1 by exponentially small terms as $\varepsilon \rightarrow 0$, we must insert a boundary-layer near $\partial \Omega$ of exponentially small amplitude. In terms of the local normaltangential coordinates $(\eta, s)$, where $\eta=\sigma / \varepsilon^{2}$, we seek a boundary-layer solution $u(\eta, s)$ of 3.1, valid near $\partial \Omega$, which behaves like (2.8) away from the layer. With this boundary-layer scaling and (2.9), (3.1) becomes

$$
\varepsilon^{2}\left[\varepsilon^{-4} u_{\eta \eta}-\varepsilon^{-2} \frac{\kappa}{1-\kappa \varepsilon^{2} \eta} u_{\eta}+\frac{1}{1-\kappa \varepsilon^{2} \eta} \frac{\partial}{\partial s}\left(\frac{1}{1-\kappa \varepsilon^{2} \eta} u_{s}\right)\right]+u \log u=0 .
$$

Then, introducing $w(\eta, s)$ by the WKB-type transformation $u(\eta, s)=\exp (w(\eta, s))$, we find that $w$ satisfies

$$
\varepsilon^{-2}\left(w_{\eta \eta}+w_{\eta}^{2}\right)-\frac{\kappa}{1-\kappa \varepsilon^{2} \eta} w_{\eta}+\frac{\varepsilon^{2}}{\left(1-\kappa \varepsilon^{2} \eta\right)^{2}}\left(w_{s s}+w_{s}^{2}\right)+\frac{\varepsilon^{4} \kappa^{\prime} \eta w_{s}}{\left(1-\kappa \varepsilon^{2} \eta\right)^{3}}+w=0 .
$$

From the matching condition 2.8 we require that the solution $w$ to 3.3 has the far-field behavior

$$
\left.w(\eta, s)\right|_{\eta \rightarrow \infty} \rightarrow-\frac{D}{4 \varepsilon^{2}}+1+\eta \chi-\frac{\varepsilon^{2} \eta^{2}}{4} .
$$

This limiting behavior suggests that we seek a solution to 3.3 in the form

$$
w_{B}(\eta, s)=-\frac{D}{4 \varepsilon^{2}}+w_{0}(\eta, s)+\varepsilon^{2} w_{1}(\eta, s)+\cdots .
$$

Notice that by combining (1.4) and $u=\exp w$ it follows that $w_{B}$ is directy proportional to the flame-front interface $S$. Hence, the expansion (3.5) is essentially an expansion of the interface $S$. By substituting (3.5) into (3.3), and collecting terms of order $O\left(\varepsilon^{-2}\right)$, we find that $w_{0}(\eta, s)$ satisfies

$$
w_{0 \eta \eta}+w_{0 \eta}^{2}=\chi^{2}, \quad 0 \leqslant \eta<\infty ;\left.\quad w_{0 \eta}\right|_{\eta=0}=0,\left.\quad w_{0}\right|_{\eta \rightarrow \infty} \sim 1+\eta \chi .
$$

Similarly, from the $O(1)$ terms, we obtain the following problem for $w_{1}(\eta, s)$ :

$$
\begin{gathered}
w_{1 \eta \eta}+2 w_{0 \eta} w_{1 \eta}=\kappa w_{0 \eta}-w_{0}+\frac{1}{4} D^{\prime \prime}+\frac{1}{2} D^{\prime} w_{0 s}-\frac{1}{8} \kappa \eta\left(D^{\prime}\right)^{2}, \quad 0 \leqslant \eta<\infty, \\
\left.w_{1 \eta}\right|_{\eta=0}=0,\left.\quad w_{1}\right|_{\eta \rightarrow \infty} \sim-\frac{\eta^{2}}{4}+o(1) .
\end{gathered}
$$

The resulting boundary-layer solution $u_{B}$ of 3.1 is then given by

$$
u_{B}(\eta, s)=e^{w_{B}(\eta, s)}=\exp \left(-\frac{D}{4 \varepsilon^{2}}+w_{0}(\eta, s)+\varepsilon^{2} w_{1}(\eta, s)+\cdots\right) .
$$


The problem (3.6) is equivalent to equation (2.9) of [14] for the one-dimensional case. The solution is

$$
w_{0}(\eta, s)=\log \left[2 e^{1} \cosh (\eta \chi)\right]=1+\eta \chi+w_{0 p},
$$

where $w_{0 p}(\eta, s)$ is defined by

$$
w_{0 p}=\log \left[1+e^{-2 \chi \eta}\right] \sim e^{-2 \chi \eta} \rightarrow 0 \quad \text { as } \eta \rightarrow \infty .
$$

For a convex domain $\chi=\mathbf{d} \cdot \mathbf{e}_{\sigma} / 2>0$, and hence $w_{0 p}=O\left(e^{-2 \chi \eta}\right)$ decays exponentially as $\eta \rightarrow \infty$.

Next we solve 3.7 for $w_{1}$ in the form $w_{1}(\eta, s)=-\eta^{2} / 4+w_{1 p}(\eta, s)$. From 3.7a), we find that $w_{1 p}$ satisfies

$$
\mathrm{L} w_{1 p} \equiv w_{1 p \eta \eta}+2 w_{0 \eta} w_{1 p \eta}=(\kappa+\eta) w_{0 \eta}-w_{0}+\frac{1}{2}+\frac{1}{4} D^{\prime \prime}+\frac{1}{2} D^{\prime} w_{0 s}-\frac{1}{8} \kappa \eta\left(D^{\prime}\right)^{2} .
$$

We then substitute $3.9 \mathrm{a}$ into 3.10 ) to get

$$
\mathrm{L} w_{1 p}=(\kappa+\eta) w_{0 p \eta}-w_{0 p}+\frac{1}{2} D^{\prime} w_{0 p s}+\left[-\frac{1}{2}+\kappa \chi+\frac{1}{4} D^{\prime \prime}\right]+\eta\left[\frac{1}{2} D^{\prime} \chi^{\prime}-\frac{1}{8} \kappa\left(D^{\prime}\right)^{2}\right] .
$$

On the right-hand side of (3.11) the primes indicate differentiation with respect to $s$.

Since $w_{1}=-\eta^{2} / 4+w_{1 p}$, a sufficient condition for the far-field behavior $w_{1} \sim-\eta^{2} / 4+o(1)$ as $\eta \rightarrow \infty$ is that $w_{1 p}$ decays exponentially as $\eta \rightarrow \infty$. Since $w_{0 \eta} \sim \chi$ as $\eta \rightarrow \infty$ it follows from 3.11 that this exponential decay condition holds provided that the right-hand side of 3.11 vanishes as $\eta \rightarrow \infty$. To show this, we first use (3.9b) to conclude that the terms on the right-hand side of 3.11) that involve $w_{0 p}$ decay exponentially as $\eta \rightarrow \infty$. Next, we use 2.6 to calculate $D^{\prime}=-2 \mathbf{e}_{s} \cdot \mathbf{d}$ and $D^{\prime \prime}=2-2 \kappa \mathbf{e}_{\sigma} \cdot \mathbf{d}$. With $2 \chi=\mathbf{d} \cdot \mathbf{e}_{\sigma}$, we get

$$
-\frac{1}{2}+\kappa \chi+\frac{1}{4} D^{\prime \prime}=-\frac{1}{2}+\kappa \chi+\frac{1}{4}(2-4 \kappa \chi)=0 .
$$

By using the definition of the curvature $\kappa$ in 2.5, the last bracket on the right-hand side of (3.11) also vanishes:

$$
\eta\left[\frac{1}{2} D^{\prime} \chi^{\prime}-\frac{1}{8} \kappa\left(D^{\prime}\right)^{2}\right]=\frac{1}{2} \eta\left[-\left(\mathbf{d} \cdot \mathbf{e}_{s}\right)\left(\mathbf{d} \cdot \mathbf{e}_{\sigma}\right)^{\prime}-\kappa\left(\mathbf{d} \cdot \mathbf{e}_{s}\right)^{2}\right]=\frac{1}{2} \eta\left(\mathbf{d} \cdot \mathbf{e}_{s}\right)\left(\mathbf{d} \cdot\left(-\mathbf{e}_{\sigma}^{\prime}-\kappa \mathbf{e}_{s}\right)\right)=0 .
$$

From 3.12, 3.13, and the decay of $w_{0 p}$ as $\eta \rightarrow \infty$, we conclude that the right-hand side 3.11 decays as $\eta \rightarrow \infty$. Therefore, $w_{1 p}$ has exponential decay as $\eta \rightarrow \infty$.

For the eigenvalue estimate of $\S 3.1$, we require an explicit formula for $\gamma$ defined by

$$
\left.\gamma \equiv w_{1 p}(\eta, s)\right|_{\eta=0}=\left.\left(w_{1}(\eta, s)+\frac{\eta^{2}}{4}\right)\right|_{\eta=0}=\left.w_{1}(\eta, s)\right|_{\eta=0} .
$$

By substituting (3.12) and (3.13) into (3.11) we find that $w_{1 p}$ satisfies

$$
\begin{aligned}
& \mathrm{L} w_{1 p}=(\kappa+\eta) w_{0 p \eta}-w_{0 p}+\frac{1}{2} D^{\prime} w_{0 p s}, \quad 0 \leqslant \eta<\infty, \\
& \left.w_{1 p \eta}\right|_{\eta=0}=0, \quad w_{1 p} \text { and } w_{1 p \eta} \rightarrow 0 \quad \text { as } \eta \rightarrow \infty .
\end{aligned}
$$


To determine $\gamma$ it is convenient to introduce the adjoint problem for $h(\eta, s)$ defined by

$$
\mathrm{L}^{\dagger} h=h_{\eta \eta}-\left(2 w_{0 \eta} h\right)_{\eta}=0, \quad 0 \leqslant \eta<\infty ; \quad h \rightarrow 1 \text { and } h_{\eta} \rightarrow 0 \quad \text { as } \eta \rightarrow \infty .
$$

By using 3.9a for $w_{0}$, the solution to 3.16 is readily calculated as $h=1+e^{-2 \chi \eta}$.

Next, we use Lagrange's identity between $\mathrm{L}$ and $\mathrm{L}^{\dagger}$ to obtain

$$
\int_{0}^{\infty} h \mathrm{~L} w_{1 p} \mathrm{~d} \eta=\left.\left(h w_{1 p \eta}-h_{\eta} w_{1 p}\right)\right|_{0} ^{\infty}+\left.2 w_{0 \eta} h w_{1 p}\right|_{0} ^{\infty} .
$$

Then, by using $\left.w_{0 \eta}\right|_{\eta=0}=\left.w_{1 p \eta}\right|_{\eta=0}=0,\left.h_{\eta}\right|_{\eta=0}=-2 \chi$, together with the decay of $w_{1 p}$ and $w_{1 p \eta}$ as $\eta \rightarrow \infty, 3.17$ and 3.15 determine $\gamma$ as

$$
\begin{aligned}
\gamma & =\left.w_{1 p}\right|_{\eta=0}=-\frac{1}{2 \chi} \int_{0}^{\infty} h \mathrm{~L} w_{1 p} \mathrm{~d} \eta \\
& =-\frac{1}{2 \chi} \int_{0}^{\infty}\left(1+e^{-2 \chi \eta}\right)\left[(\kappa+\eta) w_{0 p \eta}-w_{0 p}+\frac{1}{2} D^{\prime} w_{0 p s}\right] \mathrm{d} \eta .
\end{aligned}
$$

From 3.9b, 3.18 becomes

$$
\begin{aligned}
\gamma & =-\frac{1}{2 \chi} \int_{0}^{\infty}\left(1+e^{-2 \chi \eta}\right)\left[\frac{-2 \chi(\kappa+\eta)}{1+e^{-2 \chi \eta}} e^{-2 \chi \eta}-\log \left(1+e^{-2 \chi \eta}\right)-\frac{\chi^{\prime} D^{\prime} \eta}{1+e^{-2 \chi \eta}} e^{-2 \chi \eta}\right] \mathrm{d} \eta \\
& =\frac{1}{2 \chi} \int_{0}^{\infty}\left[2(\kappa+\eta) \chi e^{-2 \chi \eta}+\left(1+e^{-2 \chi \eta}\right) \log \left(1+e^{-2 \chi \eta}\right)+\eta \kappa\left(\mathbf{d} \cdot \mathbf{e}_{s}\right)^{2} e^{-2 \chi \eta}\right] \mathrm{d} \eta .
\end{aligned}
$$

In obtaining the last line above we used the relation $D^{\prime} \chi^{\prime}=\kappa\left(\mathbf{d} \cdot \mathbf{e}_{s}\right)^{2}$ found in 3.13 . Finally, we split the integral above into three separate terms as

$$
\begin{aligned}
2 \chi \gamma= & \left.\int_{0}^{\infty}\left(1+e^{-2 \chi \eta}\right) \log \left(1+e^{-2 \chi \eta}\right) d \eta+\int_{0}^{\infty} \eta\left[2 \chi+\kappa\left(\mathbf{d} \cdot \mathbf{e}_{s}\right)^{2}\right)\right] e^{-2 \chi \eta} \mathrm{d} \eta \\
& +\int_{0}^{\infty} 2 \kappa \chi e^{-2 \chi \eta} \mathrm{d} \eta .
\end{aligned}
$$

Each of the integrals above is readily evaluated. In this way, we obtain the explicit result

$$
\gamma=\left.w_{1}\right|_{\eta=0}=\left.w_{1 p}\right|_{\eta=0}=\frac{\kappa}{2 \chi}+\frac{1}{(2 \chi)^{2}}\left(\frac{\pi^{2}}{12}+2 \log 2+\frac{\kappa\left(\mathbf{e}_{s} \cdot \mathbf{d}\right)^{2}}{2 \chi}\right) .
$$

This result is then used in (3.8) to calculate $u_{B}$ on $\partial \Omega$. By using $\left.w_{0}\right|_{\eta=0}=1+\log 2$ and 3.20) we conclude that

$$
\left.u_{B}\right|_{\partial \Omega}=\left.e^{w_{B}}\right|_{\eta=0} \sim 2 \exp \left(-\frac{D}{4 \varepsilon^{2}}+1\right)\left(1+\varepsilon^{2} \gamma+\cdots\right) .
$$

In summary, for $\mathbf{x} \in \Omega$, the quasi-equilibrium solution, denoted by $u_{\varepsilon}\left(\mathbf{x} ; \mathbf{x}_{0}\right)$, has the form

$$
u_{\varepsilon}\left(\mathbf{x} ; \mathbf{x}_{0}\right) \equiv \begin{cases}u^{\infty}\left(\mathbf{x} ; \mathbf{x}_{0}\right) \equiv \exp \left(1-\frac{\left|\mathbf{x}-\mathbf{x}_{0}\right|^{2}}{4 \varepsilon^{2}}\right), & \operatorname{dist}(\mathbf{x}, \partial \Omega) \gg O\left(\varepsilon^{2}\right), \\ u_{B}=\exp \left(-\frac{D}{4 \varepsilon^{2}}+w_{0}+\varepsilon^{2} w_{1}\right), & \operatorname{dist}(\mathbf{x}, \partial \Omega)=O\left(\varepsilon^{2}\right) .\end{cases}
$$


By adding the outer and boundary-layer solutions, and then subtracting their common parts, one can, in the usual way, obtain a uniformly valid representation for the quasi-equilibrium solution.

Consider the special case where $\Omega$ is the unit disk. Let $\mathbf{x}_{0}=r_{0}\left(\cos \theta_{0}, \sin \theta_{0}\right)$ denote the spike location in $\Omega$ with $0 \leqslant r_{0}<1$. In terms of polar coordinates, $\partial \Omega$ is parameterized as $\mathbf{x}(\theta)=$ $(\cos \theta, \sin \theta)$. We calculate

$$
\begin{aligned}
\mathbf{e}_{s} \cdot \mathbf{d} & =(-\sin \theta, \cos \theta) \cdot\left(r_{0} \cos \theta_{0}-\cos \theta, r_{0} \sin \theta_{0}-\sin \theta\right)=-r_{0} \sin \left(\theta-\theta_{0}\right), \\
\mathbf{e}_{\sigma} \cdot \mathbf{d} & =-(\cos \theta, \sin \theta) \cdot\left(r_{0} \cos \theta_{0}-\cos \theta, r_{0} \sin \theta_{0}-\sin \theta\right)=1-r_{0} \cos \left(\theta-\theta_{0}\right) .
\end{aligned}
$$

Therefore, with $\kappa=1$ and 3.23 , the expression for $\gamma=\left.w_{1}\right|_{\eta=0}$ in the boundary estimate 3.21 , for $u_{B}$ becomes

$$
\gamma=\frac{1}{2 \chi}+\frac{1}{(2 \chi)^{2}}\left(\frac{\pi^{2}}{12}+2 \log 2+\frac{r_{0}^{2} \sin ^{2}\left(\theta-\theta_{0}\right)}{2 \chi}\right), \quad 2 \chi=1-r_{0} \cos \left(\theta-\theta_{0}\right) .
$$

\subsection{The critical eigenfunctions and eigenvalues}

We now derive precise asymptotic estimates for the two critical eigenfunctions and eigenvalues of (3.2). To derive the eigenvalue estimates we must first determine asymptotic formulae for the critical eigenfunctions on the boundary of the domain. As in 2.10) of $\$ 2$ we denote by $\phi^{\infty}$ one of the two translation eigenfunctions for the infinite-domain problem. We look for an eigenfunction of (3.2) in the additive boundary-layer form of (2.11). By substituting (2.11) into (3.2), and assuming that $\lambda$ is exponentially small, we obtain

$$
\begin{aligned}
\varepsilon^{2} \Delta \phi_{B}+\left(1+\log u_{\varepsilon}\right) \phi_{B} & =-\left[\varepsilon^{2} \Delta \phi^{\infty}+\left(1+\log u^{\infty}\right) \phi^{\infty}\right]+\phi^{\infty}\left(\log u^{\infty}-\log u_{\varepsilon}\right) \\
& =\phi^{\infty}\left(\log u^{\infty}-\log u_{\varepsilon}\right) .
\end{aligned}
$$

In (3.25) we noted that the first term in the middle expression of (3.25) vanishes identically. Within an $O\left(\varepsilon^{2}\right)$ neighborhood of $\partial \Omega$ we replace $u_{\varepsilon}$ by the boundary-layer function $u_{B}$ of 3.8 ) to find that $\phi_{B}$ satisfies

$$
\begin{aligned}
& \varepsilon^{2} \Delta \phi_{B}+\left(1+\log u_{B}\right) \phi_{B}=\phi^{\infty}\left(\log u^{\infty}-\log u_{B}\right) \equiv R, \quad \mathbf{x} \in \Omega \\
& \partial_{\eta} \phi_{B}=-\varepsilon^{2} \partial_{\sigma} \phi^{\infty}, \quad \mathbf{x} \in \partial \Omega .
\end{aligned}
$$

The boundary data in 3.26 was calculated in (2.14) and $u_{B}$ was given in (3.8). In this way, we obtain

$$
\begin{gathered}
\varepsilon^{2} \Delta \phi_{B}+\left(1-\frac{D}{4 \varepsilon^{2}}+w_{0}+\varepsilon^{2} w_{1}\right) \phi_{B}=R \equiv-\phi^{\infty}\left(w_{0 p}+\varepsilon^{2} w_{1 p}+\cdots\right), \\
\partial_{\eta} \phi_{B}=-\frac{1}{2}\left(d_{i} \chi-\varepsilon^{2} \mathbf{e}_{i} \cdot \mathbf{e}_{\sigma}\right) \exp \left(1-\frac{D}{4 \varepsilon^{2}}\right) \quad \text { on } \eta=0 .
\end{gathered}
$$

Here $w_{0 p}$ and $w_{1 p}$ satisfy $(3.9 \mathrm{~b})$ and $(3.15)$, respectively.

To show that $\phi_{B} \rightarrow 0$ as $\eta \rightarrow \infty$ away from the boundary-layer region, it suffices to show that $R$ decays to zero as $\eta \rightarrow \infty$. In the far-field, 2.13) shows that $\phi^{\infty}=O\left(e^{\chi \eta}\right)$, while from (3.9b) and 3.15 we get $\left(w_{0 p}+\varepsilon^{2} w_{1 p}\right)=O\left(\varepsilon^{q} e^{-2 \chi^{\eta}}\right)$. Therefore, $R=O\left(\varepsilon^{q} e^{-\chi \eta}\right) \rightarrow 0$ as $\eta \rightarrow \infty$. 
Next, we seek a solution to (3.27) in the form (2.16). We then substitute (2.16) into (3.27) after first expressing the Laplacian in (3.27a) in terms of normal-tangential coordinates as in 2.9). Upon collecting the lowest powers of $\varepsilon$, we find that $\Phi_{B 0}$ satisfies

$$
\left(\Phi_{B 0}\right)_{\eta \eta}-\chi^{2} \Phi_{B 0}=0, \quad 0 \leqslant \eta<\infty ;\left.\quad\left(\Phi_{B 0}\right)_{\eta}\right|_{\eta=0}=-d_{i} \chi / 2,\left.\quad \Phi_{B 0}\right|_{\eta \rightarrow \infty} \rightarrow 0 .
$$

In terms of $w_{0}$ and $w_{0 p}$ defined in (3.9), we find at next order that $\Phi_{B 1}$, on $0 \leqslant \eta<\infty$, satisfies

$$
\begin{aligned}
&\left(\Phi_{B 1}\right)_{\eta \eta}-\chi^{2} \Phi_{B 1}= \kappa\left(\Phi_{B 0}\right)_{\eta}+\frac{1}{2} D^{\prime}\left(\Phi_{B 0}\right)_{s} \\
&+\Phi_{B 0}\left(\frac{1}{4} D^{\prime \prime}-\frac{1}{8} \kappa \eta\left(D^{\prime}\right)^{2}-1-w_{0}\right)-\frac{1}{2} d_{i} e^{\chi \eta} w_{0 p}, \\
&\left.\left(\Phi_{B 1}\right)_{\eta}\right|_{\eta=0}=\frac{1}{2} \mathbf{e}_{i} \cdot \mathbf{e}_{\sigma},\left.\quad \Phi_{B 1}\right|_{\eta \rightarrow \infty} \rightarrow 0 .
\end{aligned}
$$

The solution to $(3.28)$ is simply

$$
\Phi_{B 0}=\frac{d_{i}}{2} e^{-\chi \eta}
$$

We then substitute (3.30) and $w_{0}=1+\chi \eta+w_{0 p}$ (see (3.9) ) into the right-hand side of 3.29a, and we use the following identities to simplify the resulting expressions:

$$
\frac{D}{4}-\frac{\left(D^{\prime}\right)^{2}}{16}=\chi^{2}, \quad D^{\prime}=-2 \mathbf{e}_{s} \cdot \mathbf{d}, \quad D^{\prime \prime}=2-4 \kappa \chi, \quad \chi^{\prime}=-\frac{\kappa}{2} \mathbf{e}_{s} \cdot \mathbf{d} .
$$

In this way, we find after some algebra that $\Phi_{B 1}$ satisfies

$$
\mathrm{L} \Phi_{B 1}=C(\eta, s) \equiv-\Phi_{B 0}\left[2 \kappa \chi+\frac{3}{2}+\chi \eta+\kappa \eta\left(\mathbf{d} \cdot \mathbf{e}_{s}\right)^{2}+\left(\mathbf{d} \cdot \mathbf{e}_{s}\right) \frac{d_{i}^{\prime}}{d_{i}}+\left(1+e^{2 \chi \eta}\right) w_{0 p}\right],
$$

with the boundary condition given in $3.29 \mathrm{~b}$. In 3.32 , we have introduced the self-adjoint operator $\mathrm{L}=\partial_{\eta}^{2}-\chi^{2}$. Since $w_{0 p}=O\left(e^{-2 \chi \eta}\right)($ see $\sqrt{3.9})$ and $\Phi_{B 0}=O\left(e^{-\chi \eta}\right)$ as $\eta \rightarrow \infty$, it follows that $C(\eta, s)=O\left(\eta e^{-\chi \eta}\right)$ as $\eta \rightarrow \infty$.

Below we require an estimate for $\Phi_{B}$ on the boundary. Therefore, from the solution to 3.32 we must calculate $\beta=\left.\Phi_{B 1}\right|_{\eta=0}$. To do so, we use a similar procedure as in $\S 2$ that avoids having to calculate the entire function $\Phi_{B 1}(\eta, s)$ directly. Let $h(\eta, s)$ satisfy the adjoint problem

$$
\mathrm{L} h=h_{\eta \eta}-\chi^{2} h=0, \quad 0 \leqslant \eta<\infty ;\left.\quad h\right|_{\eta=0}=1,\left.\quad h_{\eta}\right|_{\eta=0}=-\chi .
$$

The solution is $h=e^{-\chi \eta}$. Then, by using 3.33, 3.32, and 3.29b, together with the Lagrange identity

$$
\int_{0}^{\infty} h \mathrm{~L} \Phi_{B 1} \mathrm{~d} \eta-\int_{0}^{\infty} \Phi_{B 1} \mathrm{~L} h \mathrm{~d} \eta=\left.\left[h\left(\Phi_{B 1}\right)_{\eta}-\Phi_{B 1} h_{\eta}\right]\right|_{0} ^{\infty},
$$

we can readily determine $\beta$ in terms of a quadrature as

$$
\beta=-\frac{1}{2 \chi} \mathbf{e}_{i} \cdot \mathbf{e}_{\sigma}-\frac{1}{\chi} \int_{0}^{\infty} e^{-\chi \eta} C(\eta, s) \mathrm{d} \eta .
$$


Next, we substitute $(3.32$ ) for $C$ into $(3.35)$ and we decompose the resulting expression into three readily evaluated integrals as in 3.19 . In this way, we obtain

$$
\beta=\left.\Phi_{B 1}\right|_{\eta=0}=-\frac{1}{2 \chi} \mathbf{e}_{i} \cdot \mathbf{e}_{\sigma}+\frac{d_{i}}{2 \chi}\left[\kappa+\frac{1}{\chi}\left(\frac{\pi^{2}}{24}+\log 2+\frac{1}{2}+\left(\mathbf{d} \cdot \mathbf{e}_{s}\right) \frac{d_{i}^{\prime}}{2 d_{i}}\right)+\frac{\kappa}{4 \chi^{2}}\left(\mathbf{d} \cdot \mathbf{e}_{s}\right)^{2}\right] .
$$

Finally, by combining (2.13), 2.11, 2.16, 3.30 , and 3.36 , we obtain the following estimate for the critical eigenfunctions of the finite-domain eigenvalue problem 3.2) on the boundary of the domain:

$$
\left.\phi\right|_{\eta=0} \sim \exp \left(1-\frac{D}{4 \varepsilon^{2}}\right)\left(d_{j}+\varepsilon^{2} \beta+\cdots\right) .
$$

Here $d_{i}(s)=x_{0 i}-x_{i}(s), D(s)=\left|\mathbf{x}(s)-\mathbf{x}_{0}\right|^{2}$, and $\beta$ is given in $(3.36)$.

Consider the special case where $\Omega$ is the unit disk with a spike at $\mathbf{x}_{0}=r_{0}\left(\cos \theta_{0}, \sin \theta_{0}\right)$ with $0 \leqslant r_{0}<1$. Then the polar angle $\theta$ denotes arclength, $D(\theta)=\left|\mathbf{x}(\theta)-\mathbf{x}_{0}\right|^{2}=1-2 r_{0} \cos \left(\theta-\theta_{0}\right)+r_{0}^{2}$, and $2 \chi=1-r_{0} \cos \left(\theta-\theta_{0}\right)$. Since $\kappa=1$, it follows from 3.23) that $\beta$ in 3.37) and 3.36 can be written as

$$
\beta=-\frac{1}{2 \chi} \mathbf{e}_{i} \cdot \mathbf{e}_{\sigma}+\frac{d_{i}}{2 \chi}\left[1+\frac{1}{\chi}\left(\frac{\pi^{2}}{24}+\log 2+\frac{1}{2}-r_{0} \sin \left(\theta-\theta_{0}\right) \frac{d_{i}^{\prime}}{2 d_{i}}\right)+\frac{r_{0}^{2}}{4 \chi^{2}} \sin ^{2}\left(\theta-\theta_{0}\right)\right] .
$$

Next, we estimate the two exponentially small eigenvalues for 3.2 corresponding to the neartranslation eigenfunctions. By using Green's identity on 3.2) we get

$$
\int_{\Omega}\left[\partial_{i} u_{\varepsilon} \mathcal{L}_{\varepsilon} \phi-\phi \mathcal{L}_{\varepsilon}\left(\partial_{i} u_{\varepsilon}\right)\right] \mathrm{d} \mathbf{x}=\int_{\partial \Omega} \varepsilon^{2}\left[\partial_{i} u_{\varepsilon} \partial_{n} \phi-\phi \partial_{n}\left(\partial_{i} u_{\varepsilon}\right)\right] \mathrm{d} s .
$$

Then, since $\mathcal{L}_{\varepsilon} \phi=\lambda \phi$ and $\partial_{n} \phi=0$ on $\partial \Omega, 3.39$ becomes

$$
\lambda \int_{\Omega} \phi \partial_{i} u_{\varepsilon} \mathrm{d} \mathbf{x}=\int_{\Omega} \phi \mathcal{L}_{\varepsilon}\left(\partial_{i} u_{\varepsilon}\right) \mathrm{d} \mathbf{x}-\varepsilon^{2} \int_{\partial \Omega} \phi \partial_{n}\left[\partial_{i} u_{\varepsilon}\right] \mathrm{d} s .
$$

The dominant contribution to the integral multiplying $\lambda$ occurs from the region near the spike where $u_{\varepsilon} \sim u^{\infty}$ and $\phi \sim \varepsilon^{2} \partial_{i} u^{\infty}$ from 1.10 and 2.10, respectively. In addition, since $u_{\varepsilon}=u_{B}$ on $\partial \Omega$ from 3.8, 3.40 becomes

$$
\lambda \mathcal{J} \sim-\mathcal{I}+\mathcal{K}
$$

where $\mathcal{J}, \mathcal{I}$, and $\mathcal{K}$ are defined by

$$
\mathcal{J} \equiv \varepsilon^{2} \int_{\Omega}\left(\partial_{i} u^{\infty}\right)^{2} d \mathbf{x}, \quad \mathcal{I} \equiv \varepsilon^{2} \int_{\partial \Omega} \phi \partial_{n}\left[\partial_{i} u_{B}\right] \mathrm{d} s, \quad \mathcal{K} \equiv \int_{\Omega} \phi \mathcal{L}_{\varepsilon}\left(\partial_{i} u_{\varepsilon}\right) \mathrm{d} \mathbf{x} .
$$

The integral $\mathcal{J}$ was estimated in $2.24 \mathrm{p}$. We will estimate $\mathcal{I}$ precisely as $\varepsilon \rightarrow 0$. In Appendix A we show that $\mathcal{K}$ is asymptotically smaller than $\mathcal{I}$ as $\varepsilon \rightarrow 0$, and therefore can be neglected. Hence, 3.41a) reduces to $\lambda \mathcal{J} \sim-\mathcal{I}$.

To calculate $\mathcal{I}$ we first evaluate $\partial_{i} u_{B}$ as

$$
\partial_{i} u_{B}=\mathbf{e}_{i} \cdot \nabla u_{B}=\mathbf{e}_{i} \cdot\left(\mathbf{e}_{s} \partial_{s} u_{B}+\mathbf{e}_{\sigma} \partial_{\sigma} u_{B}\right)=\left(\mathbf{e}_{i} \cdot \mathbf{e}_{s}\right) \partial_{s} u_{B}+\varepsilon^{-2}\left(\mathbf{e}_{i} \cdot \mathbf{e}_{\sigma}\right) \partial_{\eta} u_{B} .
$$

Therefore, since $\mathbf{e}_{i} \cdot \mathbf{e}_{s}$ and $\mathbf{e}_{i} \cdot \mathbf{e}_{\sigma}$ depend only on $s$, we further calculate on $\partial \Omega$ that

$$
-\partial_{n}\left(\partial_{i} u_{B}\right)=\partial_{\sigma}\left(\partial_{i} u_{B}\right)=\varepsilon^{-2}\left(\mathbf{e}_{i} \cdot \mathbf{e}_{s}\right) \partial_{s \eta} u_{B}+\varepsilon^{-4}\left(\mathbf{e}_{i} \cdot \mathbf{e}_{\sigma}\right) \partial_{\eta \eta} u_{B} .
$$


Next, since $\partial_{s \eta} u_{B}=0$ on $\eta=0$ and $u_{B}=e^{w_{B}}$ from 3.8, 3.43) becomes

$$
\left.\partial_{n}\left[\partial_{i} u_{B}\right]\right|_{\eta=0}=-\left.\varepsilon^{-4}\left(\mathbf{e}_{i} \cdot \mathbf{e}_{\sigma}\right) \partial_{\eta \eta} u_{B}\right|_{\eta=0}=-\left.\varepsilon^{-4}\left(\mathbf{e}_{i} \cdot \mathbf{e}_{\sigma}\right) u_{B} \partial_{\eta \eta} w_{B}\right|_{\eta=0}
$$

In (3.21) we have an estimate for $u_{B}$ on $\eta=0$. Hence, we need only estimate $\left.\partial_{\eta \eta} w_{B}\right|_{\eta=0}$. By using 3.5 for $w_{B}$, together with $3.9 \mathrm{a}, w_{1}=-\eta^{2} / 4+w_{1 p}$, and 3.15 , we obtain

$$
\left.\partial_{\eta \eta} w_{B}\right|_{\eta=0}=\left.\left[w_{0 \eta \eta}+\varepsilon^{2}\left(w_{1 p}-\eta^{2} / 4\right)_{\eta \eta}+\cdots\right]\right|_{\eta=0}=\chi^{2}-\varepsilon^{2}\left(\frac{1}{2}+\log 2+\kappa \chi\right)+\cdots .
$$

Therefore, substituting 3.21 and 3.45 into 3.44, we obtain

$$
\left.\partial_{n}\left[\partial_{i} u_{B}\right]\right|_{\eta=0}=-2 \varepsilon^{-4}\left(\mathbf{e}_{i} \cdot \mathbf{e}_{\sigma}\right) \exp \left(1-\frac{D}{4 \varepsilon^{2}}\right)\left[\chi^{2}+\varepsilon^{2}\left(\chi^{2} \gamma-\frac{1}{2}-\log 2-\kappa \chi\right)+\cdots\right],
$$

where $\gamma$ is defined in 3.20). Then, by substituting (3.46, and 3.37) for $\phi$, into 3.41b for $\mathcal{I}$, we conclude

$$
\mathcal{I} \sim-2 \varepsilon^{-2} e^{2} \int_{\partial \Omega} e^{-D /\left(2 \varepsilon^{2}\right)}\left(\mathbf{e}_{i} \cdot \mathbf{e}_{\sigma}\right)\left(d_{i}+\varepsilon^{2} \beta\right)\left[\chi^{2}+\varepsilon^{2}\left(\gamma \chi^{2}-\frac{1}{2}-\log 2-\kappa \chi\right)\right] \mathrm{d} s .
$$

Finally, we substitute 3.36 and 3.20 for $\beta$ and $\gamma$, respectively, into 3.47). This gives our final estimate

$$
\mathcal{I} \sim-2 \varepsilon^{-2} e^{2} \int_{\partial \Omega} e^{-D /\left(2 \varepsilon^{2}\right)}\left[\mathcal{F}_{0}(s)+\varepsilon^{2} \mathcal{F}_{1}(s)+\cdots\right] \mathrm{d} s,
$$

where $\mathcal{F}_{0}$ and $\mathcal{F}_{1}$ are defined by

$$
\begin{aligned}
& \mathcal{F}_{0}(s) \equiv d_{i} \chi^{2}\left(\mathbf{e}_{i} \cdot \mathbf{e}_{\sigma}\right), \\
& \mathcal{F}_{1}(s) \equiv\left(-\frac{\chi}{2} \mathbf{e}_{i} \cdot \mathbf{e}_{\sigma}+\frac{d_{i}}{4}\left[\frac{\pi^{2}}{6}-1+\frac{\kappa}{\chi}\left(\mathbf{e}_{s} \cdot \mathbf{d}\right)^{2}+\left(\mathbf{e}_{s} \cdot \mathbf{d}\right) \frac{d_{i}^{\prime}}{d_{i}}\right]\right)\left(\mathbf{e}_{i} \cdot \mathbf{e}_{\sigma}\right) .
\end{aligned}
$$

Finally, by substituting 3.48 and 2.24 into 3.41a, we obtain the following result:

PRincipal Result 3.1 Let $\mathcal{F}_{0}$ and $\mathcal{F}_{1}$ be as defined in $3.48 \mathrm{~b}$. Then, for $\varepsilon \rightarrow 0$, the two exponentially small eigenvalues of (3.2) corresponding to the near-translation eigenfunctions have the asymptotic estimate

$$
\lambda \sim \frac{4 \varepsilon^{-4}}{\pi} \int_{\partial \Omega} e^{-D /\left(2 \varepsilon^{2}\right)} \mathcal{F}_{\varepsilon}(s) \mathrm{d} s, \quad \mathcal{F}_{\varepsilon}(s) \equiv \mathcal{F}_{0}(s)+\varepsilon^{2} \mathcal{F}_{1}(s)+\cdots .
$$

Next, we use Laplace's method (cf. [24]) to asymptotically evaluate the integral in 3.49 for $\varepsilon \ll 1$. For $\varepsilon \rightarrow 0$, the dominant contribution to this integral arises from the point $s_{0}$ on $\partial \Omega$ closest to the spike location $\mathbf{x}_{0}$. Assume that there is only one such point where $D(s)$ takes its global minimum for $s \in \partial \Omega$. Then, since $\chi^{2}=D / 4$ at $s=s_{0}$ from 3.31 , we find that $\mathcal{F}_{0}=\left(\mathbf{e}_{i} \cdot \mathbf{e}_{\sigma}\right) d_{i} D / 4$ at $s=s_{0}$. Therefore, Laplace's method on 3.49 gives the following leading-order estimate for the two exponentially small eigenvalues:

$$
\lambda \sim \frac{2 \varepsilon^{-3}}{\left(\pi D_{0}^{\prime \prime}\right)^{1 / 2}} d_{i 0} D_{0}\left(\mathbf{e}_{i} \cdot \mathbf{e}_{\sigma}\right) \exp \left(-\frac{D_{0}}{2 \varepsilon^{2}}\right), \quad D_{0}^{\prime \prime}=2-2 \kappa_{0}\left|\mathbf{d}_{0}\right|
$$


In 3.50$), D_{0} \equiv\left|\mathbf{x}\left(s_{0}\right)-\mathbf{x}_{0}\right|^{2},\left|\mathbf{d}_{0}\right| \equiv \sqrt{D_{0}}, d_{i 0} \equiv x_{0 i}-x_{i}\left(s_{0}\right)$, and $\kappa_{0}$ is the curvature of $\partial \Omega$ at $s=s_{0}$.

The result 3.50) for 3.2), based on linearizing (1.8) around a spike profile with boundary layer, is of the same asymptotic order as the corresponding result 2.27) for 2.4 obtained by linearizing (1.8) solely around the spike profile $u^{\infty}$. However, the pre-exponential factors in these two estimates are slightly different. In Appendix B we retain higher-order terms in the asymptotic evaluation of 3.49 to obtain the following more precise result:

PRINCIPAL RESUlt 3.2 Assume that there is a unique point $s_{0}$ on $\partial \Omega$ where $D(s)$ is minimized. Then, for $\varepsilon \rightarrow 0$, the two exponentially small eigenvalues of 3.2 corresponding to the neartranslation eigenfunctions satisfy

$$
\lambda \sim \frac{8 \varepsilon^{-3}}{\sqrt{\pi D_{0}^{\prime \prime}}} e^{-D_{0} /\left(2 \varepsilon^{2}\right)}\left[\mathcal{F}_{00}+\varepsilon^{2}\left(\mathcal{F}_{10}-\mathcal{F}_{00}^{\prime} \frac{D_{0}^{\prime \prime \prime}}{\left(D_{0}^{\prime \prime}\right)^{2}}+\frac{\mathcal{F}_{00}^{\prime \prime}}{D_{0}^{\prime \prime}}\right)\right] .
$$

Here we have labeled $\left.\mathcal{F}_{00}^{(k)} \equiv \mathcal{F}_{0}^{(k)}\right|_{s=s_{0}}$ for $k \geqslant 1,\left.\mathcal{F}_{j 0} \equiv \mathcal{F}_{j}\right|_{s=s_{0}}$ for $j=0,1$, and $\left.D_{0}^{(k)} \equiv D^{(k)}\right|_{s=s_{0}}$ for $k \geqslant 1$. The various terms in 3.51) are given explicitly by

$$
\begin{gathered}
\mathcal{F}_{00}=\frac{d_{i 0} D_{0}}{4}\left(\mathbf{e}_{i} \cdot \mathbf{e}_{\sigma}\right), \quad \mathcal{F}_{10}=\left(-\frac{\sqrt{D_{0}}}{4} \mathbf{e}_{i} \cdot \mathbf{e}_{\sigma}+\frac{d_{i 0}}{4}\left(\frac{\pi^{2}}{6}-1\right)\right)\left(\mathbf{e}_{i} \cdot \mathbf{e}_{\sigma}\right), \\
\mathcal{F}_{00}^{\prime}=\frac{D_{0}}{4}\left[d_{i 0}^{\prime}\left(\mathbf{e}_{i} \cdot \mathbf{e}_{\sigma}\right)-d_{i 0} \kappa_{0}\left(\mathbf{e}_{i} \cdot \mathbf{e}_{s}\right)\right], \quad D_{0}^{\prime \prime}=2-2 \kappa_{0}\left|\mathbf{d}_{0}\right|, \quad D_{0}^{\prime \prime \prime}=-2 \kappa_{0}^{\prime}\left|\mathbf{d}_{0}\right|, \\
\mathcal{F}_{00}^{\prime \prime}=-\frac{D_{0}}{4}\left(\mathbf{e}_{i} \cdot \mathbf{e}_{s}\right)\left[2 d_{i 0}^{\prime} \kappa_{0}+d_{i 0} \kappa_{0}^{\prime}\right]+\frac{\left(\mathbf{e}_{i} \cdot \mathbf{e}_{\sigma}\right)}{4}\left[D_{0} d_{i 0}^{\prime \prime}+2 \sqrt{D_{0}} \kappa_{0} d_{i 0}-3 D_{0} d_{i 0} \kappa_{0}^{2}\right] .
\end{gathered}
$$

We now illustrate the result 3.51 for the exponentially small eigenvalues of 3.2 for two particular domains. Firstly, let $\Omega$ be the square $[0,3] \times[0,3]$ with a spike at $\mathbf{x}_{0}=(2.0,0.8)$. Then $(2.0,0.0)$ is the unique point on $\partial \Omega$ closest to $\mathbf{x}_{0}$ and $\left|\mathbf{d}_{0}\right|=d_{20}=0.8$. We set $i=2$ in (3.52), corresponding to the near-translation eigenvalue in the $x_{2}$ direction, and use $\mathbf{e}_{2} \cdot \mathbf{e}_{\sigma}=1$, $\kappa_{0}=0$ and $d_{20}^{\prime}=0$ in $3.52 \mathrm{a}$, 3.52b, and 3.52c, to get

$$
\mathcal{F}_{00}=\frac{d_{20}^{3}}{4}, \quad \mathcal{F}_{10}=-\frac{d_{20}}{4}+\frac{d_{20}}{4}\left(\frac{\pi^{2}}{6}-1\right), \quad \mathcal{F}_{00}^{\prime}=0, \quad \mathcal{F}_{00}^{\prime \prime}=0 .
$$

Then from (3.51) we obtain the following asymptotic estimate for the translation eigenvalue in the $x_{2}$ direction:

$$
\lambda \sim \sqrt{\frac{2}{\pi}} \varepsilon^{-3} d_{20}^{3} e^{-d_{20}^{2} /\left(2 \varepsilon^{2}\right)}\left[1+\frac{\varepsilon^{2}}{d_{20}^{2}}\left(\frac{\pi^{2}}{6}-2\right)+\cdots\right] .
$$

This result is equivalent to that in equation (3.28) of [14] for the one-dimensional slab geometry.

Secondly, we consider the unit disk with a spike centered at $\mathbf{x}_{0}=(\mu, 0)$, with $0<\mu<1$. Then $(1,0)$ is the unique point on $\partial \Omega$ closest to $\mathbf{x}_{0}$, with minimum distance $-d_{10}=1-\mu>0$. We consider the near-translation eigenvalue in the $x_{1}$ direction. From 3.52a), 3.52b), and 3.52c), with $\mathbf{e}_{1}=(1,0)$ and $\mathbf{e}_{\sigma}=(-1,0)$, we calculate

$$
\begin{gathered}
\mathcal{F}_{00}=\frac{\left|d_{10}\right|^{3}}{4}, \quad \mathcal{F}_{10}=\frac{\left|d_{10}\right|}{4}\left(\frac{\pi^{2}}{6}-2\right), \quad \mathcal{F}_{00}^{\prime}=0, \quad \mathcal{F}_{00}^{\prime \prime}=\left[\frac{d_{10}^{2}}{4}-\frac{3\left|d_{10}\right|^{3}}{4}\right], \\
D_{0}^{\prime \prime}=2-2\left|d_{10}\right|, \quad D_{0}^{\prime \prime \prime}=0 .
\end{gathered}
$$


In calculating $\mathcal{F}_{00}^{\prime \prime}$ we used $d_{10}^{\prime \prime}=1$ as found by parameterizing $\partial \Omega$ by polar coordinates. Upon substituting (3.55) into 3.51, we obtain the following asymptotic estimate for the near-translation eigenvalue in the $x_{1}$ direction:

$$
\lambda \sim \sqrt{\frac{2}{\pi\left(1-\left|d_{10}\right|\right)}} \varepsilon^{-3}\left|d_{10}\right|^{3} e^{-d_{10}^{2} /\left(2 \varepsilon^{2}\right)}\left[1+\frac{\varepsilon^{2}}{d_{10}^{2}}\left(\frac{\pi^{2}}{6}-2+\frac{\left|d_{10}\right|-3 d_{10}^{2}}{2\left(1-\left|d_{10}\right|\right)}\right)+\cdots\right] .
$$

\section{Metastable flame-front dynamics}

In order to explicitly characterize the metastable behavior for 1.1 , in this section we derive an asymptotic ODE for the location $\mathbf{x}_{0}(t)$ of the tip of the paraboloidal flame-front. We do not analyze the initial formation of a flame-front interface from arbitrary initial data. Instead, we analyze the slow motion of the flame-front after it has formed from initial data. Hence, we look for a solution to (1.5) in the form

$$
\mathcal{U}=e^{c} e^{\omega}\left(u^{\infty}+E\right),
$$

where $u^{\infty} \equiv u^{\infty}\left[\mathbf{x} ; \mathbf{x}_{0}(t)\right]$ is the spike profile of 1.10 . Here $c=c(t)$ with $c^{\prime}=O\left(\varepsilon^{-2}\right) \gg 1$ determines the speed of the flame-front, whereas $\omega(t)$ and $\mathbf{x}_{0}(t)$ are assumed to be slowly varying functions of $t$. The initial condition is taken to be $\mathcal{U}(\mathbf{x}, 0)=u^{\infty}\left[\mathbf{x} ; \mathbf{x}_{0}(0)\right]$ with $\mathbf{x}_{0}(0) \in \Omega$ and $\operatorname{dist}\left(\mathbf{x}_{0}(0), \partial \Omega\right)=O(1)$ as $\varepsilon \rightarrow 0$. Therefore, we take $c=\omega=0$ at $t=0$, and $E(\mathbf{x}, 0)=0$. The error term $E=E(\mathbf{x}, t)$ is required to satisfy $E \ll u^{\infty}$ with $\omega^{\prime}=O(E)$. The condition that $E$ remain small over exponentially long time intervals will determine explicit ordinary differential equations governing the dynamics of the slow growth $\omega(t)$ and of the flame-front tip $\mathbf{x}_{0}(t)$.

We begin by substituting (4.1) into $(1.5)$ to obtain

$$
\begin{aligned}
\left(c^{\prime}+\omega^{\prime}\right)\left(u^{\infty}+E\right) & +\left(u_{t}^{\infty}+E_{t}\right) \\
& =\varepsilon^{2}\left(\Delta u^{\infty}+\Delta E\right)+\left(u^{\infty}+E\right)\left[\log \left(u^{\infty}+E\right)-\left\langle\log \left(u^{\infty}+E\right)\right\rangle\right]
\end{aligned}
$$

In calculating the right-hand side of 4.2 we retain the linear terms in the error $E$ and we use the equation 1.9 for $u^{\infty}$. On the left-hand side of 4.2 we neglect the quadratically small term $\omega^{\prime} E$. In this way, we get

$$
c^{\prime} u^{\infty}+c^{\prime} E+\omega^{\prime} u^{\infty}+u_{t}^{\infty}+E_{t}=\mathcal{L}_{0} E-u^{\infty}\left(\left\langle\log u^{\infty}\right\rangle+\left\langle E / u^{\infty}\right\rangle\right)-E\left\langle\log u^{\infty}\right\rangle .
$$

Here the operator $\mathcal{L}_{0}$ is defined in 2.4. We then choose $c^{\prime}$, with $c(0)=0$, by

$$
c^{\prime}=-\left\langle\log u^{\infty}\right\rangle-\left\langle E / u^{\infty}\right\rangle
$$

Upon substituting (4.4) into 4.3, and neglecting the quadratic term in $E$, we find that $E$ satisfies

$$
\begin{array}{ll}
E_{t}=\mathcal{L}_{0} E-\omega^{\prime} u^{\infty}-u_{t}^{\infty}, & \mathbf{x} \in \Omega, t>0 ; \\
\partial_{n} E=-\partial_{n} u^{\infty}, \quad \mathbf{x} \in \partial \Omega ; & E(\mathbf{x}, 0)=0 .
\end{array}
$$

In $\S 2$ the largest three eigenvalues $\lambda_{j}$, for $j=0,1,2$, of $\mathcal{L}_{0}$ were calculated asymptotically for $\varepsilon \rightarrow 0$. The remaining eigenvalues $\lambda_{j}$ for $j \geqslant 3$ of $\mathcal{L}_{0}$, representing decaying modes, are strictly negative for $\varepsilon \rightarrow 0$ and are asymptotically close to the negative eigenvalues of the 2-D harmonic oscillator 2.3. The principal eigenvalue of $\mathcal{L}_{0}$ is $\lambda_{0} \sim 1$, with $\phi_{0} \sim M_{0} u^{\infty}$ away from $\partial \Omega$. An 
estimate for $\lambda_{0}-1$ was given in Principal Result 2.2. The two exponentially small near-translation eigenvalues $\lambda_{j}$, for $j=1,2$, of $\mathcal{L}_{0}$, with $\phi_{j} \sim M_{j} \varepsilon^{2} \partial_{i} u^{\infty}$ away from $\partial \Omega$, were calculated in Principal Result 2.1. Here $M_{j}$ is a normalization constant. We order the remaining eigenvalues as $\lambda_{j+1} \leqslant \lambda_{j}$ for $j \geqslant 3$, and we expand $E$ in terms of the normalized eigenfunctions $\phi_{j}$ of $\mathcal{L}_{0}$ as

$$
E(\mathbf{x}, t)=\sum_{j=0}^{\infty} b_{j}(t) \phi_{j}(\mathbf{x}), \quad\left(\phi_{j}, \phi_{j}\right)=1
$$

Here we have defined the inner product $(u, v) \equiv \int_{\Omega} u v \mathrm{~d} \mathbf{x}$. By using Green's identity on 4.5 , together with the properties $\mathcal{L}_{0} \phi_{j}=\lambda_{j} \phi_{j}$ and $\partial_{n} \phi_{j}=0$ on $\partial \Omega$, we readily derive the following initial-value problem for $b_{j}(t)$ :

$$
\begin{aligned}
b_{j}^{\prime}-\lambda_{j} b_{j} & =\mathcal{R}_{j} \equiv-\varepsilon^{2} \int_{\partial \Omega} \phi_{j} \partial_{n} u^{\infty} \mathrm{d} s-\omega^{\prime}\left(u^{\infty}, \phi_{j}\right)-\left(u_{t}^{\infty}, \phi_{j}\right), \\
b_{j}(0) & =0, \quad j=0,1, \ldots
\end{aligned}
$$

Since $\lambda_{0}>0$ and $\lambda_{j}$ is exponentially small for $j=1,2$, we must impose that $\mathcal{R}_{j}=0$ for $j=0,1,2$ in order to ensure that the coefficients $b_{j}(t)$ for $j \geqslant 0$, and hence $E(\mathbf{x}, t)$, are small over exponentially long time intervals. Therefore, $\omega^{\prime}$ and $\mathbf{x}_{0}^{\prime}$ are to be found from

$$
\omega^{\prime}\left(u^{\infty}, \phi_{j}\right)+\left(u_{t}^{\infty}, \phi_{j}\right)=-\varepsilon^{2} \int_{\partial \Omega} \phi_{j} \partial_{n} u^{\infty} \mathrm{d} s, \quad j=0,1,2 .
$$

We remark that the structure in (4.7), in which an extra degree of freedom, representing the $\omega^{\prime}$ term, is needed to eliminate growth on a fast time-scale due to a strictly positive eigenvalue, also occurred in $\S 4$ of [17] in analyzing the metastable motion of a bubble solution for a nonlocal mass-conserving Allen-Cahn equation.

The system (4.8) for $\mathbf{x}_{0}(t)$ and $\omega(t)$ asymptotically decouples for $\varepsilon \ll 1$. We set $j=0$ in 4.8 and use $\phi_{0} \sim u^{\infty}$ in the inner products. Since $\left(u_{t}^{\infty}, \phi_{0}\right)$ is the dot product of $\mathbf{x}_{0}^{\prime}$ and an exponentially small inner product, we obtain

$$
\omega^{\prime}\left(u^{\infty}, u^{\infty}\right) \sim-\varepsilon^{2} \int_{\partial \Omega} \phi_{0} \partial_{n} u^{\infty} \mathrm{d} s .
$$

The boundary integral on the right-hand side of 4.9 can be expressed in terms of $\lambda_{0}-1$. To see this, we use Green's identity on [2.4], together with $\mathcal{L}_{0} u^{\infty}=u^{\infty}$, to get

$$
\left(u^{\infty}, \mathcal{L}_{0} \phi_{0}\right)-\left(\phi_{0}, \mathcal{L}_{0} u^{\infty}\right)=\left(\lambda_{0}-1\right)\left(u^{\infty}, \phi_{0}\right)=-\varepsilon^{2} \int_{\partial \Omega} \phi_{0} \partial_{n} u^{\infty} \mathrm{d} s .
$$

Upon substituting (4.10) into 4.9 , and using $\left(u^{\infty}, \phi_{0}\right) \sim\left(u^{\infty}, u^{\infty}\right)$, we obtain the explicit ODE $\omega^{\prime}(t) \sim \lambda_{0}-1$ with $\omega(0)=0$. In Principal Result 2.2 it was shown that $\lambda_{0}-1$ is exponentially small for $\varepsilon \rightarrow 0$ for any $\mathbf{x}_{0} \in \Omega$. Therefore, if we write $\lambda_{0}=\lambda_{0}\left[\mathbf{x}_{0}(t)\right]$, we conclude that $\omega^{\prime}$ is exponentially small and that

$$
\omega(t) \sim \int_{0}^{t}\left(\lambda_{0}\left[\mathbf{x}_{0}(\tau)\right]-1\right) \mathrm{d} \tau
$$


To determine the ODE for $\mathbf{x}_{0}(t)$, we set $j=1,2$ in 4.8 and use $\phi_{j} \sim \varepsilon^{2} \partial_{j} u^{\infty}$ to estimate the inner products in 4.8 . For $j=1,2$ the term $\omega^{\prime}\left(u^{\infty}, \phi_{j}\right)$ is the product of two exponentially small terms and can be neglected. Therefore, (4.8) reduces to

$$
\left(u_{t}^{\infty}, \phi_{j}\right) \sim-\varepsilon^{2} \int_{\partial \Omega} \phi_{j} \partial_{n} u^{\infty} \mathrm{d} s, \quad j=1,2
$$

To evaluate the left-hand side of 4.12 we use $\phi_{j} \sim \varepsilon^{2} \partial_{j} u^{\infty}=\frac{1}{2}\left(x_{0 j}-x_{j}\right) u^{\infty}$ from 2.13), and we differentiate 1.10 with respect to $t$. Evaluating the resulting integral we obtain

$$
\begin{aligned}
\left(u_{t}^{\infty}, \phi_{j}\right) & \sim-\frac{x_{0 j}^{\prime}}{4 \varepsilon^{2}} \int_{\Omega}\left(x_{j}-x_{0 j}\right)^{2}\left(u^{\infty}\right)^{2} \mathrm{~d} \mathbf{x} \sim-\frac{\pi e^{2} x_{0 j}^{\prime}}{4 \varepsilon^{2}} \int_{0}^{\infty} \rho^{3} e^{-\rho^{2} /\left(2 \varepsilon^{2}\right)} \mathrm{d} \rho \\
& =-\frac{\pi}{2} \varepsilon^{2} e^{2} x_{0 j}^{\prime} .
\end{aligned}
$$

To evaluate the boundary integral on the right-hand side of (4.12) we use the formulae 2.20 and 2.31 for $\phi_{j}$ and $\partial_{n} u^{\infty}$ on $\partial \Omega$, respectively. This gives

$$
-\varepsilon^{2} \int_{\partial \Omega} \phi_{j} \partial_{n} u^{\infty} \mathrm{d} s=\frac{e^{2}}{2} \int_{\partial \Omega} D e^{-D /\left(2 \varepsilon^{2}\right)}\left(\hat{\mathbf{d}} \cdot \mathbf{e}_{\sigma}\right) \hat{d}_{j} \mathrm{~d} s
$$

Here $\hat{\mathbf{d}}$ is the unit vector in the direction of $\mathbf{d}$ and $\hat{d}_{j}$ is its $j^{\text {th }}$ component. By substituting 4.13 , and (4.14) into 4.12, we obtain an ODE for $\mathbf{x}_{0}(t)$. The flame-front interface $S(\mathbf{x}, t)$ is obtained by substituting (4.1) into (1.4), and using (1.10), (4.4), and (4.11), for $u^{\infty}, c$, and $\omega$, respectively. In this way, we obtain the following explicit characterization of the metastable flame-front dynamics for $[1.1]$ :

PRINCIPAL RESUlt 4.1 For $\varepsilon \rightarrow 0$, the outer approximation for the flame-front interface $S(\mathbf{x}, t)$ of 1.1 , valid away from $\partial \Omega$, is

$$
S(\mathbf{x}, t) \sim-\frac{\left|\mathbf{x}-\mathbf{x}_{0}(t)\right|^{2}}{2}+\frac{1}{2} \int_{0}^{t}\left\langle\left|\mathbf{x}-\mathbf{x}_{0}(\tau)\right|^{2}\right\rangle \mathrm{d} \tau+2 \varepsilon^{2}\left[1-t+\int_{0}^{t}\left(\lambda_{0}\left[\mathbf{x}_{0}(\tau)\right]-1\right) \mathrm{d} \tau\right] .
$$

The flame-front tip $\mathbf{x}_{0}(t)$ and the flame-front speed speed $v(t) \equiv 2 \varepsilon^{2} c^{\prime}(t)$ satisfy

$$
\mathbf{x}_{0}^{\prime} \sim \mathbf{I}\left(\mathbf{x}_{0}\right) \equiv-\frac{1}{\pi \varepsilon^{2}} \int_{\partial \Omega} D e^{-D /\left(2 \varepsilon^{2}\right)}\left(\hat{\mathbf{d}} \cdot \mathbf{e}_{\sigma}\right) \hat{\mathbf{d}} \mathrm{d} s, \quad v(t)=\frac{1}{2}\left\langle\left|\mathbf{x}-\mathbf{x}_{0}(t)\right|^{2}\right\rangle-2 \varepsilon^{2} .
$$

Here $D(s) \equiv\left|\mathbf{x}_{0}-\mathbf{x}(s)\right|^{2}, \hat{\mathbf{d}}=\left(\mathbf{x}_{0}-\mathbf{x}(s)\right) /\left|\mathbf{x}_{0}-\mathbf{x}(s)\right|$, and $\mathbf{e}_{\sigma}$ is the inward pointing unit normal to $\partial \Omega$. Suppose that at $t=0$ there is a unique point $\mathbf{x}\left(s_{0}\right)$ on $\partial \Omega$ that is closest to $\mathbf{x}_{0}(0)$. Then, the motion of $\mathbf{x}_{0}(t)$ is towards the same closest boundary point $\mathbf{x}\left(s_{0}\right)$ for all subsequent time, and the distance $d_{0}(t) \equiv\left|\mathbf{x}_{0}(t)-\mathbf{x}\left(s_{0}\right)\right|$ to $\partial \Omega$ satisfies

$$
d_{0}^{\prime} \sim-\sqrt{\frac{2}{\pi}} \frac{d_{0}^{2}}{\varepsilon \sqrt{1-\kappa_{0} d_{0}}} e^{-d_{0}^{2} /\left(2 \varepsilon^{2}\right)},
$$

with initial value $d_{0}(0)=\left|\mathbf{x}_{0}(0)-\mathbf{x}\left(s_{0}\right)\right|$. Here $\kappa_{0}$ is the curvature of $\partial \Omega$ at $s=s_{0}$. 
The result in 4.17) follows by using Laplace's method on (4.16) to derive

$$
\mathbf{x}_{0}^{\prime} \sim-\sqrt{\frac{2}{\pi}} \frac{D_{0}}{\varepsilon \sqrt{1-\kappa_{0} d_{0}}} e^{-D_{0} /\left(2 \varepsilon^{2}\right)} \hat{\mathbf{d}}_{0} .
$$

where $D_{0}=D\left(s_{0}\right), d_{0}=\sqrt{D_{0}}$, and $\hat{\mathbf{d}}_{0}$ is a unit vector in the direction of $\mathbf{x}_{0}-\mathbf{x}\left(s_{0}\right)$. The result 4.17 then follows readily from 4.18 by taking the dot product with $\hat{\mathbf{d}}_{0}$.

We now make a few remarks. Firstly, the condition $\mathbf{I}\left(\mathbf{x}_{0}\right)=0$ determines the unstable equilibrium point $\mathbf{x}_{0 e}$ for $\mathbf{x}_{0}(t)$. A similar condition involving the vanishing of a vector boundary integral was obtained in [18] for spike-layer solutions of $\varepsilon^{2} \Delta u-u+u^{2}=0$ in $\Omega \in \mathbb{R}^{2}$. For a strictly convex domain, the analysis in $\S 3$ of [18] can be adapted to show that $\mathbf{x}_{0 e}$ is $O(\varepsilon)$ close to the center $\mathbf{x}_{i n}$ of the unique largest inscribed circle for $\Omega$. Since the calculation of the $O(\varepsilon)$ correction term is similar to that in $\S 3$ of [18] we do not pursue the details here.

Secondly, we remark that the ODE (4.17) for the two-dimensional case is remarkably similar to the ODE (1.2) for the one-dimensional slab geometry. Specifically, upon retaining only one of the two exponential terms in 1.2 , the only difference between these two ODE's is the curvature term in 4.17). The primary reason for this similarity is that the analytical form of the spike profile satisfying (1.9) does not exhibit geometric spreading in two dimensions. In fact, for $N=1,2$ dimensions, we have $u^{\infty}=e^{N / 2} \exp \left(-\left|\mathbf{x}-\mathbf{x}_{0}\right|^{2} /\left(4 \varepsilon^{2}\right)\right)$. In contrast, for the shadow Gierer-Meinhardt model analyzed in [6], the spike profile $w(\rho)$ is the radial symmetric solution in $\mathbb{R}^{2}$ of $\Delta w-w+w^{2}=0$. This solution exhibits geometric spreading in dimension $N$ owing to the far-field behavior $w(\rho) \sim$ $a_{N} \rho^{(1-N) / 2} e^{-\rho}$ as $\rho \rightarrow \infty$ for some constant $a_{N}$. The resulting ODE for the slow motion of the spike as found in Corollary 2 of [6] was $d_{0}^{\prime} \sim-c_{\varepsilon, N} d_{0}^{(1-N) / 2}\left(1-d_{0} \kappa_{0}\right)^{-1 / 2} \exp \left(-2 d_{0} / \varepsilon\right)$ for some constant $c_{\varepsilon_{N}}$. In contrast to the results (4.17) and (1.2) for the tip of the flame-front, the pre-exponential factor in this ODE of [6] does depend significantly on the dimension $N$.

Thirdly, we remark that the pre-exponential factor of $d_{0}^{2}$ in the ODE 4.17 precludes the vanishing of $d_{0}$ in finite time. Although the ODE (4.18) is not valid when $d_{0}=O(\varepsilon)$, its extrapolation into this regime suggests that, ultimately, $d_{0}^{\prime} \sim-c d_{0}^{2}$, which decays algebraically in time. It is an open problem to analyze exactly how the flame-front interface attaches to the boundary of the domain. Finally, by separating variables in (4.17), the time $T$ for the flame-front tip to become within an $O(\varepsilon)$ distance from $\partial \Omega$ is given asymptotically by

$$
T \sim \sqrt{\frac{\pi}{2}} \frac{\varepsilon^{3} \sqrt{1-\kappa_{0} d_{00}}}{d_{00}^{3}} \exp \left[d_{00}^{2} /\left(2 \varepsilon^{2}\right)\right], \quad d_{00} \equiv d_{0}(0) .
$$

We now give a few examples to illustrate Principal Result 4.1.

EXAMPLE 4.1 Our first example is for a square domain $\Omega=[0,3] \times[0,3]$ with the flame-front tip initially at $\mathbf{x}_{0}(0)=(2.0,0.5)$. Then $(2.0,0.0)$ is the unique point on $\partial \Omega$ closest to $\mathbf{x}_{0}(0)$. From (4.17) with $\kappa_{0}=0$, the vertical distance $d_{0}(t)$ to the boundary satisfies

$$
d_{0}^{\prime} \sim-\sqrt{\frac{2}{\pi}} \frac{d_{0}^{2}}{\varepsilon} e^{-d_{0}^{2} /\left(2 \varepsilon^{2}\right)}, \quad d_{0}(0)=0.5 .
$$

In Fig. 4 we compare the solution of the ODE $(4.20)$ for $d_{0}(t)$ with corresponding results obtained from the numerical solution of the full PDE initial-boundary value problem (1.8) with the initial condition in the form of a spike 1.10 located at $\mathbf{x}_{0}(0)=(2.0,0.5)$. The numerical method is 


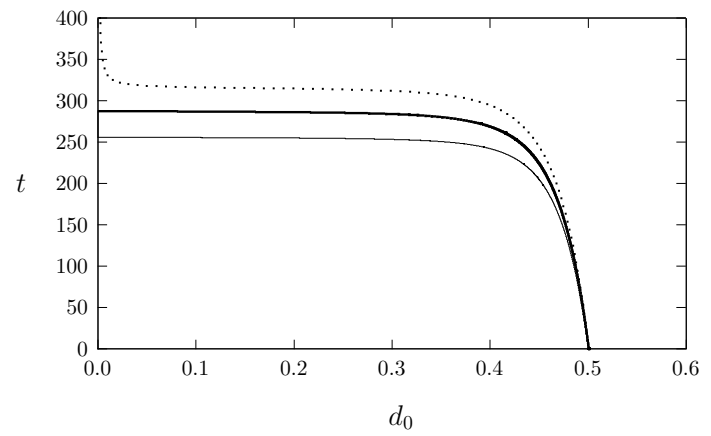

FIG. 4. The distance $d_{0}(t)$ of the flame-front tip to the boundary, from the numerical solution of the full PDE 1.8 (dashed line) and the solution of the asymptotic ODE $[4.20]$ (solid line). The domain is the square $\Omega=[0,3] \times[0,3]$ and $\varepsilon=0.113$. The heavy-solid line is the improved ODE 4.21 suggested by the one-dimensional theory.

described in Appendix C. Although the ODE (4.20) is obtained only from a leading-order analysis, reasonable agreement is present already for the relatively large value $\varepsilon=0.113$ that was used in the computations. In Fig. 4 we also plot $d_{0}(t)$ from the following ODE of equation (3.46) of [14] pertaining to a strictly one-dimensional geometry:

$$
d_{0}^{\prime} \sim-\sqrt{\frac{2}{\pi}} \frac{d_{0}^{2}}{\varepsilon} e^{-d_{0}^{2} /\left(2 \varepsilon^{2}\right)}\left(1+\frac{\pi^{2} \varepsilon^{2}}{6 d_{0}^{2}}\right), \quad d_{0}(0)=0.5 .
$$

For this ODE, $d_{0}=0$ at a finite time. Although we have not attempted to derive the $O\left(\varepsilon^{2}\right)$ coefficient in the pre-exponential factor for $d_{0}^{\prime}$ in 4.17 for an arbitrary geometry, we observe in Fig. 4 that for this special geometry the improved ODE (4.21) provides a slightly closer agreement with the full numerical results than does 4.20 .

EXAMPLE 4.2 Secondly, we let $\Omega$ be the unit disk with the flame-front tip initially located at $\mathbf{x}_{0}(0)=(0.5,0)$. Then $(1,0)$ is the unique point on $\partial \Omega$ closest to $\mathbf{x}_{0}$. By setting $\kappa_{0}=1$ in 4.17 ,

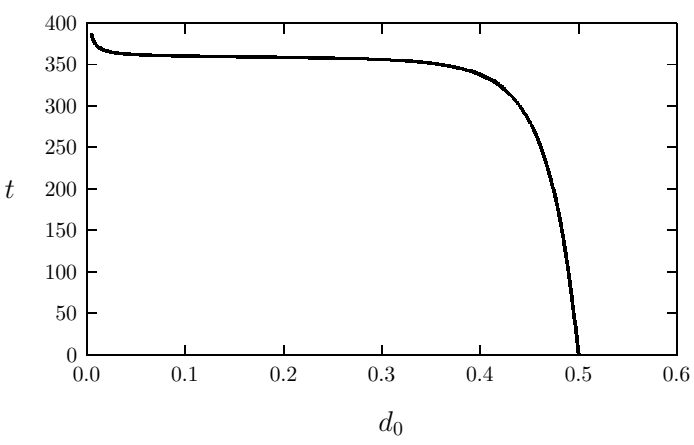

(a) $t$ versus $d_{0}$

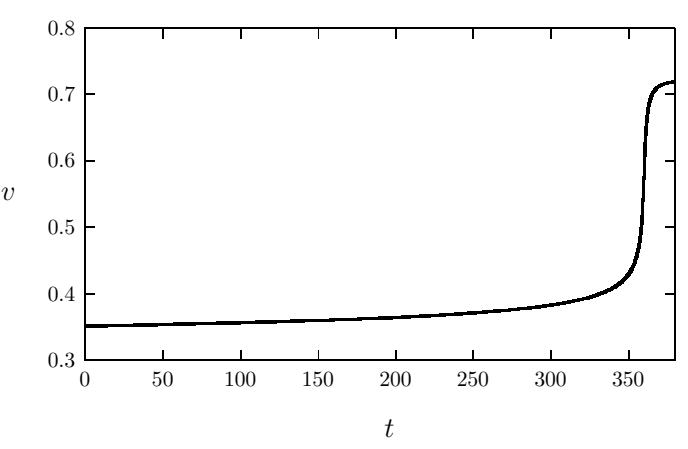

(b) $v$ versus $t$

FIG. 5. Numerical solution of 4.22 with $\varepsilon=0.11$ for the flame-front tip in the unit disk. Left: the distance $d_{0}(t)$ of the flame-front tip to the boundary. Right: the speed $v(t)$ of the flame-front. 
and with $d_{0}(0)=0.5$, we obtain the ODE for the distance $d_{0}(t)$ from the flame-front tip to the closest point $(1,0)$ on $\partial \Omega$. In terms of $d_{0}$, the speed of the flame-front interface $v$ is calculated by evaluating $\left\langle\left|\mathbf{x}-\mathbf{x}_{0}\right|^{2}\right\rangle$ explicitly in 4.16 . In this way, we get

$$
\begin{aligned}
& d_{0}^{\prime} \sim-\sqrt{\frac{2}{\pi}} \frac{d_{0}^{2}}{\varepsilon \sqrt{1-d_{0}}} e^{-d_{0}^{2} /\left(2 \varepsilon^{2}\right)}, \quad d_{0}(0)=0.5 \\
& v(t)=2 \varepsilon^{2} c^{\prime}(t)=\frac{1}{2}\left(1-d_{0}\right)^{2}+\frac{1}{4}-2 \varepsilon^{2} .
\end{aligned}
$$

In Fig. 5 we plot the numerical solution for $d_{0}(t)$ and the speed $v(t)$ when $\varepsilon=0.11$. From these figures we observe that the speed of the flame is roughly constant until the tip becomes close to the wall. Also note that $d_{0}^{\prime}$ decreases on a new time-scale when $d_{0}$ is very small, and that $d_{0}$ does not vanish in finite time.

EXAMPLE 4.3 For our third example, let $\Omega=[0,1] \times[0,1]$ contain a spike initially located at $\mathbf{x}_{0}(0)=\left(\gamma_{0}, \gamma_{0}\right)$ with $0<\gamma_{0}<1 / 2$. Then there are exactly two points on $\partial \Omega$ closest to $\mathbf{x}_{0}(0)$. Laplace's method on 4.16 then yields

$$
\mathbf{x}_{0}^{\prime} \sim-\sqrt{\frac{2}{\pi}} \frac{D_{0}}{\varepsilon} e^{-D_{0} /\left(2 \varepsilon^{2}\right)} \mathbf{e}_{1}-\sqrt{\frac{2}{\pi}} \frac{D_{0}}{\varepsilon} e^{-D_{0} /\left(2 \varepsilon^{2}\right)} \mathbf{e}_{2},
$$

where $\mathbf{e}_{j}$ is the unit vector in the $j^{\text {th }}$ direction. Here $D_{0}=\gamma^{2} / 2$, where $\gamma$ is the distance from $\mathbf{x}_{0}(t)$ to the vertex $(0,0)$ of the square. The vector addition of the two boundary forces in (4.23) shows that the flame-front tip slowly drifts towards the vertex $(0,0)$. A similar behavior was shown in [1] from full numerical solutions of 1.1$]$. From (4.23) we readily obtain the following ODE for the distance $\gamma(t)$ from the flame-front tip to the vertex $(0,0)$ :

$$
\gamma^{\prime} \sim-\frac{\gamma^{2}}{\varepsilon \sqrt{\pi}} e^{-\gamma^{2} /\left(4 \varepsilon^{2}\right)}, \quad \gamma(0)=\gamma_{0} .
$$

\section{The slow motion of a boundary spike}

The metastability analysis in $\S 4$ for 1.1 showed that the the flame-front tip inside a convex domain $\Omega$ drifts asymptotically exponentially slowly towards the closest point on the boundary $\partial \Omega$. In this section we derive an explicit asymptotic ODE for the dynamics of the flame-front tip after it has become attached to the boundary of the domain. The motion of the flame-front tip is found to be proportional to the derivative of the curvature of $\partial \Omega$, with stable rest points at local maxima of the curvature.

As in $\S 4$ we look for a solution to $(1.5)$ in the form

$$
\mathcal{U}=e^{c(t)+\omega} \mathcal{V},
$$

where $\omega$ is a slowly varying function of $t$. Upon writing the Laplacian in 1.5 in terms of the normal-tangential coordinates $(\sigma, s)$, we obtain

$$
\left(c^{\prime}+\omega^{\prime}\right) \mathcal{V}+\partial_{t} \mathcal{V}=\varepsilon^{2}\left[\mathcal{V}_{\sigma \sigma}-\frac{\kappa}{1-\kappa \sigma} \mathcal{V}_{\sigma}+\frac{1}{1-\kappa \sigma} \frac{\partial}{\partial s}\left(\frac{1}{1-\kappa \sigma} \mathcal{V}_{s}\right)\right]+\mathcal{V} \log \mathcal{V}-\mathcal{V}\langle\log \mathcal{V}\rangle
$$


We then choose $c^{\prime}=-\langle\log \mathcal{V}\rangle$ with $c(0)=0$ to eliminate the nonlocal term. Next we introduce the local normal-tangential coordinates $(\eta, \xi)$ by

$$
\eta=\varepsilon^{-1} \sigma, \quad \xi=\varepsilon^{-1}\left[s-s_{0}(\tau)\right], \quad \tau=\varepsilon^{3} t .
$$

We then expand $\mathcal{V}$ and $\omega^{\prime}$ in powers of $\varepsilon$ as

$$
\mathcal{V}=v_{0}(\eta, \xi)+\varepsilon v_{1}(\eta, \xi, t)+\varepsilon^{2} v_{2}(\eta, \xi, t)+\cdots ; \quad \omega^{\prime}=\varepsilon \omega_{0}+\varepsilon^{2} \omega_{1}+\cdots
$$

In 5.3, $s=s_{0}(\tau)$ denotes the unknown time-dependent location of the boundary spike. The choice of slow time-scale $\tau=\varepsilon^{3} t$ is a result of a solvability condition on the solution for $v_{2}$.

We substitute 5.4 with local coordinates 5.3 into 5.2 and collect powers of $\varepsilon^{0}, \varepsilon^{1}$, and $\varepsilon^{2}$. In terms of the local coordinates 5.3 the curvature $\kappa=\kappa(s)$ becomes $\kappa=\kappa_{0}+\varepsilon \xi \kappa_{0}^{\prime}+\cdots$, where $\kappa_{0} \equiv \kappa\left(s_{0}\right)$ and $\left.\kappa_{0}^{\prime} \equiv \kappa^{\prime}(s)\right|_{s=s_{0}}$. From the $O(1)$ terms we find that, on the domain $\mathbb{R}^{+} \equiv$ $\{(\xi, \eta) \mid-\infty<\xi<\infty, \eta>0\}, v_{0}$ satisfies

$$
v_{0 \xi \xi}+v_{0 \eta \eta}+Q\left(v_{0}\right)=0, \quad-\infty<\xi<\infty, 0<\eta<\infty ; \quad v_{0 \eta}=0, \quad \eta=0,
$$

where $Q\left(v_{0}\right) \equiv v_{0} \log v_{0}$. We let $(\eta, \xi)=(0,0)$ denote the boundary spike location. From 1.10 we obtain

$$
v_{0}(\xi, \eta)=\exp \left(1-\frac{\rho^{2}}{4}\right), \quad \rho^{2}=\xi^{2}+\eta^{2} .
$$

Notice that $v_{0}$ is even in $\xi$, whereas $v_{0 \xi}$ is odd in $\xi$. From collecting the $O(\varepsilon)$ and $O\left(\varepsilon^{2}\right)$ terms, we find that, on $\mathbb{R}^{+}, v_{1}$ and $v_{2}$ satisfy

$$
\begin{array}{ll}
v_{1 t}=\mathcal{L} v_{1}+\mathcal{R}_{1}, & \mathcal{R}_{1} \equiv-\omega_{0} v_{0}-\kappa_{0} v_{0 \eta}+2 \kappa_{0} \eta v_{0 \xi \xi} \\
v_{2 t}=\mathcal{L} v_{2}+\mathcal{R}_{2}, & \mathcal{R}_{2} \equiv-\omega_{0} v_{1}-\omega_{1} v_{0}+s_{0}^{\prime} v_{0 \xi}+\mathcal{F}_{e}+\mathcal{F}_{0},
\end{array}
$$

where $\mathcal{F}_{e}$ and $\mathcal{F}_{0}$ are defined by

$$
\begin{aligned}
& \mathcal{F}_{e}=-\kappa_{0}^{2} \eta v_{0 \eta}-\kappa_{0} v_{1 \eta}+3 \kappa_{0}^{2} \eta^{2} v_{0 \xi \xi}+2 \kappa_{0} \eta v_{1 \xi \xi}+\frac{v_{1}^{2}}{2} Q^{\prime \prime}\left(v_{0}\right), \\
& \mathcal{F}_{o}=\kappa_{0}^{\prime} \eta v_{0 \xi}+2 \kappa_{0}^{\prime} \eta \xi v_{0 \xi \xi}-\kappa_{0}^{\prime} \xi v_{0 \eta},
\end{aligned}
$$

with $Q^{\prime \prime}\left(v_{0}\right)=v_{0}^{-1}$. In $5.7 \mathrm{a}$ and $5.7 \mathrm{~b}$ the operator $\mathcal{L}$ is defined by

$$
\mathcal{L} \phi=\phi_{\xi \xi}+\phi_{\eta \eta}+Q^{\prime}\left(v_{0}\right) \phi
$$

where $Q^{\prime}\left(v_{0}\right)=1+\log v_{0}$. The boundary conditions for 5.7a and $5.7 \mathrm{~b}$ are that $v_{1 \eta}=v_{2 \eta}=0$ on $\eta=0$.

The spectral problem $\mathcal{L} \phi=\lambda \phi$ on $\mathbb{R}^{+}$with $\phi_{\eta}=0$ on $\eta=0$ has two nonnegative eigenvalues. These eigenpairs are $\left(\phi_{0}, \lambda_{0}\right)=\left(v_{0}, 1\right)$ and $\left(\phi_{1}, \lambda_{1}\right)=\left(v_{0 \xi}, 0\right)$. In terms of the inner product $(f, g)$ defined by

$$
(f, g) \equiv \int_{-\infty}^{\infty} \int_{0}^{\infty} f(\eta, \xi) g(\eta, \xi) \mathrm{d} \eta \mathrm{d} \xi,
$$

a necessary condition that $v_{1}$ in 5 (5.7a) tends to the steady-state solution $v_{1 h}$ satisfying $\mathcal{L} v_{1 h}=-\mathcal{R}_{1}$ as $t \rightarrow \infty$ is that $\left(\mathcal{R}_{1}, v_{0}\right)=0$ and $\left(\mathcal{R}_{1}, v_{0 \xi}\right)=0$. Since $v_{0 \xi}$ is an odd function of $\xi$, while $\mathcal{R}_{1}$ is 
even in $\xi$, it follows that $\left(\mathcal{R}_{1}, v_{0 \xi}\right)$ vanishes identically. The condition $\left(\mathcal{R}_{1}, v_{0}\right)=0$ determines $\omega_{0}$ as

$$
\omega_{0}\left(v_{0}, v_{0}\right)=-\kappa_{0}\left(v_{0}, v_{0 \eta}\right)+2 \kappa_{0}\left(v_{0}, \eta v_{0 \xi \xi}\right) .
$$

With this choice for $\omega_{0}, v_{1}$ tends to its steady-state limit $v_{1 h}$ on an asymptotically $O(1)$ time-scale. This limiting solution provides an $O(\varepsilon)$ correction to the leading-order gaussian spatial profile $v_{0}$.

We now derive an ODE for $s_{0}(\tau)$ from $5.7 \mathrm{~b}$. A necessary condition that $v_{2}$ in $5.7 \mathrm{~b}$ ) tends to its steady-state limit $v_{2 h}$ satisfying $\mathcal{L} v_{2 h}=-\mathcal{R}_{2}$ as $t \rightarrow \infty$ is that $\left(\mathcal{R}_{2}, v_{0}\right)=0$ and $\left(\mathcal{R}_{2}, v_{0 \xi}\right)=0$. The first inner product determines $\omega_{1}$, while the second inner product determines an ODE for $s_{0}(\tau)$ in the form

$$
s_{0}^{\prime}\left(v_{0 \xi}, v_{0 \xi}\right)=\omega_{0}\left(v_{1}, v_{0 \xi}\right)+\omega_{1}\left(v_{0}, v_{0 \xi}\right)-\left(\mathcal{F}_{e}, v_{0 \xi}\right)-\left(\mathcal{F}_{o}, v_{0 \xi}\right) .
$$

We substitute the steady-state limit $v_{1 h}$ for $v_{1}$, and note that $v_{1 h}$ and $\mathcal{F}_{e}$ are even in $\xi$, while $\mathcal{F}_{o}$ is odd in $\xi$. Therefore, only the last inner product on the right-hand side of (5.11) is not identically zero. Hence,

$$
s_{0}^{\prime}\left(v_{0 \xi}, v_{0 \xi}\right)=-\left(\mathcal{F}_{0}, v_{0 \xi}\right)=\kappa_{0}^{\prime}\left(\xi v_{0}, v_{0 \xi}\right)-\kappa_{0}^{\prime}\left(v_{0 \xi}, \eta v_{0 \xi}\right)-2 \kappa_{0}^{\prime}\left(v_{0 \xi}, \eta \xi v_{0 \xi \xi}\right) .
$$

We then use 5.6 to calculate the inner products in 5.12 explicitly in terms of $v_{0}(\rho)=e^{1} e^{-\rho^{2} / 4}$ as

$$
\begin{gathered}
\left(v_{0 \xi}, v_{0 \xi}\right)=\frac{\pi}{2} \int_{0}^{\infty} \rho v_{0 \rho}^{2} \mathrm{~d} p=\frac{\pi e^{2}}{4}, \quad 2\left(v_{0 \xi}, \eta \xi v_{0 \xi \xi}\right)=-\frac{2}{3} \int_{0}^{\infty} \rho^{2} v_{0 \rho}^{2} \mathrm{~d} \rho=-\frac{\sqrt{2 \pi} e^{2}}{4}, \\
\left(\xi v_{0 \eta}, v_{0 \xi}\right)=\left(\eta v_{0 \xi}, v_{0 \xi}\right)=\frac{2}{3} \int_{0}^{\infty} \rho^{2} v_{0 \rho}^{2} \mathrm{~d} \rho .
\end{gathered}
$$

Upon substituting these inner products into 5.11, and recalling that $\tau=\varepsilon^{3} t$, we obtain the following result:

PRINCIPAL RESUlt 5.1 For $\varepsilon \rightarrow 0$, the slow motion ODE for the flame-front tip $s_{0}(t)$ on the boundary of $\Omega$ is

$$
s_{0}^{\prime}(t) \sim \varepsilon^{3} \sqrt{\frac{2}{\pi}} \kappa^{\prime}\left(s_{0}\right) .
$$

Here $\kappa_{0} \equiv \kappa\left(s_{0}\right) \geqslant 0$ is the curvature of the boundary $\partial \Omega$ of the convex domain $\Omega$ at arclength coordinate $s=s_{0}$.

With initial value $s_{0}(0)$, the ODE $(5.13)$ predicts that the spike location will tend to the closest local maximum of the curvature $\kappa(s)$. Such a local maximum is a stable rest point for 5.13 . We remark that the analysis of boundary spike motion for the case where the initial point $s_{0}(0)$ is on a flat portion of the boundary of nonzero length is considerably more delicate than the analysis presented in this section. For example, such a situation arises when a flame-front becomes attached to a straight boundary of a rectangle. For such an asymptotically degenerate situation, we expect that the speed of the flame-front tip is exponentially slow and depends on the local contact behavior of the point on the boundary closest to $s_{0}(0)$ where $\kappa \neq 0$. For the shadow Gierer-Meinhardt model such an analysis was given in $\S 5$ of [7]. 


\section{Discussion}

We have given an explicit asymptotic characterization of the slow motion dynamics of the flamefront tip for (1.1). When the flame-front tip is initially inside a convex domain, we have shown that the speed of the tip is asymptotically exponentially slow as $\varepsilon \rightarrow 0$ and the motion is directed towards the closest point on the boundary of the domain. The distance to the closest point is given asymptotically by (4.17). For a flame-front attached to the boundary of the domain, the speed of the tip is algebraically slow as $\varepsilon \rightarrow 0$ and the dynamics is given asymptotically by (5.13). An open problem is to study the detailed mechanism describing the attachment of the flame-front tip to the boundary of the domain. We now illustrate the two stages of the dynamics obtained in $\$ 4$ and $\S 5$ for two specific convex domains $\Omega$.

EXAMPLE 6.1 We first consider an elliptical domain with boundary $\partial \Omega$ given by $x_{1}=2 \cos \theta$, $x_{2}=\sin \theta$, as studied in [8]. We choose $\mathbf{x}_{0}=(1.0,0.05)$ as the initial location of the flame-front tip. The domain was shown in Fig. 2(a), together with an illustration of the two distinct stages of the flame-front dynamics: the metastable stage when the tip is inside the domain, and the second stage where the tip drifts slowly along the boundary.

To characterize the metastability, we compute that the closest point to $\mathbf{x}_{0}$ on $\partial \Omega$ is $\mathbf{x} \approx$ $(0.776,0.425)$, which corresponds to $\theta \approx 0.888$. At this closest point, the curvature of $\partial \Omega$ is $\kappa_{0}=0.425$. From (4.17), the flame-front tip drifts in a straight line towards this closest point on $\partial \Omega$, and the distance $d_{0}$ to the closest point satisfies the ODE

$$
d_{0}^{\prime} \sim-\sqrt{\frac{2}{\pi}} \frac{d_{0}^{2}}{\varepsilon \sqrt{1-\kappa_{0} d_{0}}} e^{-d_{0}^{2} /\left(2 \varepsilon^{2}\right)}, \quad d_{0}(0) \approx 0.772, \quad \kappa_{0} \approx 0.425 .
$$

A plot of the numerical solution to 6.1 for $\varepsilon=0.1643$ is shown in Fig. 6(a). The ODE ceases to be valid when $d_{0}=O(\varepsilon)$, which occurs when $t \approx 740$.

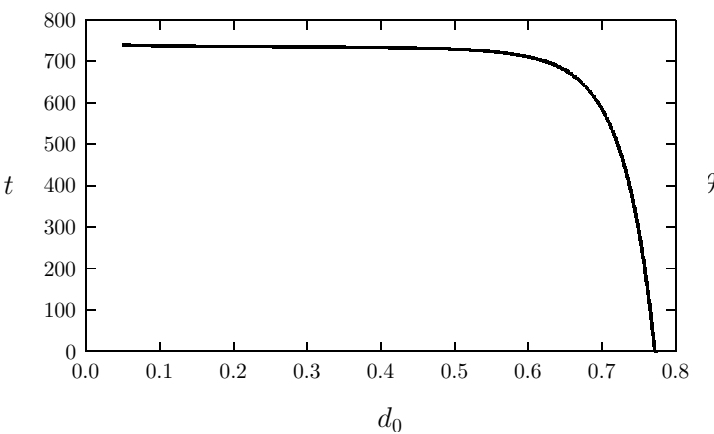

(a) $t$ versus $d_{0}$

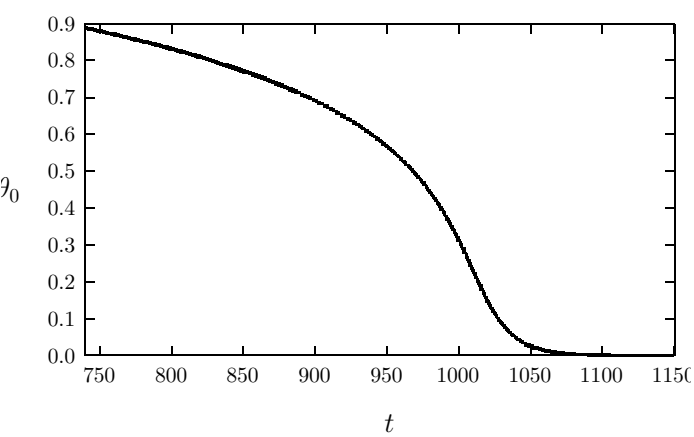

(b) $\theta_{0}$ versus $t$

FIG. 6 .

For the second stage, the motion of the flame-front tip along the boundary is given by (5.13). The mapping $s=s(\theta)$ between the arclength $s$ and the polar angle $\theta$ is chosen to be $s=\int_{0}^{\theta}[1+$ $\left.3 \sin ^{2}(\phi)\right]^{1 / 2} \mathrm{~d} \phi$. In this way, 5.13 is transformed to

$$
\theta_{0}^{\prime} \sim-9 \varepsilon^{3} \sqrt{\frac{2}{\pi}} \frac{\sin \left(2 \theta_{0}\right)}{\left[1+3 \sin ^{2}\left(\theta_{0}\right)\right]^{7 / 2}}
$$


with initial value $\theta_{0}(0) \approx 0.888$. The flame-front tip is located at $x_{0}(t)=2 \cos \left[\theta_{0}(t)\right]$ and $y_{0}(t)=$ $\sin \left[\theta_{0}(t)\right]$. Under $[6.2), \theta_{0} \rightarrow 0$ as $t \rightarrow \infty$, which corresponds to the nearest local maximum of the boundary curvature.

Finally, the upward speed $v(t)$ of the flame-front in the channel, defined in 4.16, can be determined in terms of the location $\mathbf{x}_{0}(t)$ of the flame-front tip. By calculating an area integral, we obtain

$$
v(t)=\frac{1}{2}\left\langle\left|\mathbf{x}-\mathbf{x}_{0}\right|^{2}\right\rangle-2 \varepsilon^{2}=\frac{5}{8}+\frac{1}{2}\left[x_{0}^{2}(t)+y_{0}^{2}(t)\right]-2 \varepsilon^{2} .
$$

The speed $v(t)$, computed from the asymptotic results for $x_{0}(t)$ and $y_{0}(t)$, is plotted in Fig.2(b)

This example with $\varepsilon=0.1643$ is equivalent to the example (with $\varepsilon=0.027$ as defined in [8]) studied numerically in [8]. Even with the relatively large value $\varepsilon=0.1643$, our asymptotic results, valid for $\varepsilon \ll 1$, compare reasonably well with the numerical results reported in [8].

EXAMPLE 6.2 For our second example, we let $\zeta(\theta)$ be a positive $2 \pi$-periodic function, and assume that $\zeta(\theta)+\zeta^{\prime \prime}(\theta)>0$ for $0 \leqslant \theta \leqslant 2 \pi$. Then a convex domain is generated if we define $\partial \Omega$ in parametric form as

$$
x_{1}(\theta)=\zeta(\theta) \cos \theta-\zeta^{\prime}(\theta) \sin \theta, \quad x_{2}(\theta)=\zeta(\theta) \sin \theta+\zeta^{\prime}(\theta) \cos \theta
$$

with $0 \leqslant \theta \leqslant 2 \pi$ (cf. [7]). Let $s=h(\theta)$ denote the mapping between $\theta$ and the arclength $s$. Then $f^{\prime}(\theta)$ and the curvature $\kappa(\theta)$ of the boundary are given by

$$
f^{\prime}(\theta)=\zeta(\theta)+\zeta^{\prime \prime}(\theta), \quad \kappa(\theta)=\left[\zeta(\theta)+\zeta^{\prime \prime}(\theta)\right]^{-1} .
$$

We take $\zeta(\theta)=2+\sin ^{3}(\theta)$, and we assume that the initial flame-front tip location within $\Omega$ is at $\mathbf{x}_{0}(0)=(0.5,0.0)$. A simple numerical computation shows that the closest point on $\partial \Omega$ is at $\mathbf{x} \approx(0.663,-0.944)$ corresponding to $\theta=4.883$. At this closest point the curvature is $\kappa_{0}=0.267$.

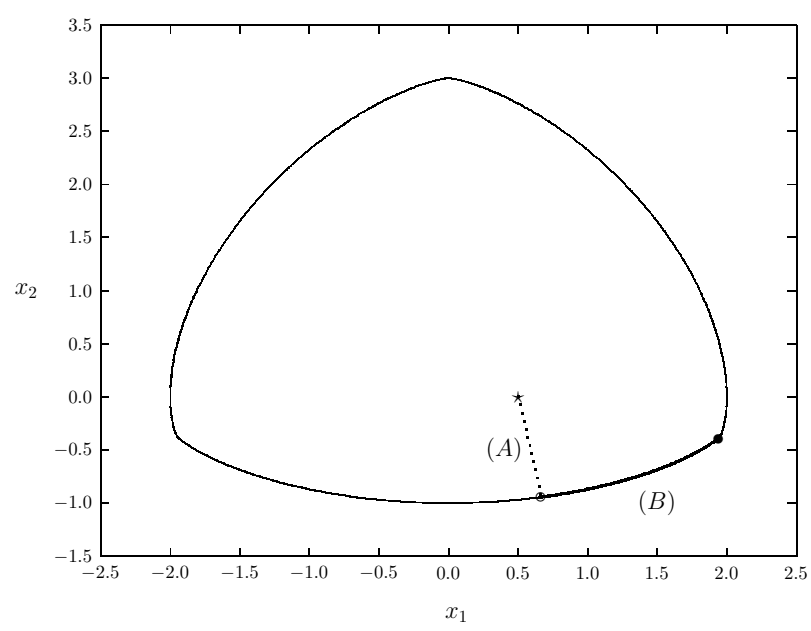

FIG. 7. Dynamics of the flame-front tip. (A) Under the dynamics 6.1 the tip moves from the initial point (labeled by $\star$ ) to an $O(\epsilon)$ neighborhood of the closest boundary point (labeled by $\circ$ ). (B) Then, under the dynamics 6.6, the tip drifts along the boundary to the nearest local maximum of the curvature (labeled by $\bullet$ ). 
From (4.17), the distance of the flame-front tip to the closest point on $\partial \Omega$ satisfies the ODE 6.1] with $\kappa_{0} \approx 0.267$ and with initial distance $d_{0}(0) \approx 0.958$. Along the boundary, we use 6.5 and (5.13) to show that the location of the flame-front tip on the boundary is given by $s_{0}(t)=f\left[\theta_{0}(t)\right]$, where $\theta_{0}(t)$ satisfies

$$
\theta_{0}^{\prime} \sim-\varepsilon^{3} \sqrt{\frac{2}{\pi}} \frac{\zeta^{\prime}\left(\theta_{0}\right)+\zeta^{\prime \prime \prime}\left(\theta_{0}\right)}{\left[\zeta\left(\theta_{0}\right)+\zeta^{\prime \prime}\left(\theta_{0}\right)\right]^{4}}
$$

Here $\zeta(\theta)=2+\sin ^{3}(\theta)$ and $\theta_{0}(0)=4.883$. The domain is shown in Fig. 7, together with an illustration of the two distinct stages of the dynamics. As shown in Fig.7 the flame-front tip on the boundary tends to the closest local maximum of the curvature.

\section{Appendix A. Asymptotic estimate for $\mathcal{K}$}

In this appendix we estimate the integral $\mathcal{K}$, defined in $3.41 \mathrm{~b}$, for $\varepsilon \rightarrow 0$. We show formally that $\mathcal{K} \ll \mathcal{I}$, where $\mathcal{I}$ is defined in $3.41 \mathrm{~b}$. Hence, we may neglect $\mathcal{K}$ in $3.41 \mathrm{a}$ ) and obtain $\lambda \mathcal{J} \sim-\mathcal{I}$.

In order to neglect $\mathcal{K}$ in comparison with the two-term approximation for $\mathcal{I}$ given in 3.48a), we must show that

$$
\mathcal{K} \equiv \int_{\Omega} \phi \mathcal{L}_{\varepsilon}\left(\partial_{i} u_{\varepsilon}\right) \mathrm{d} \mathbf{x} \ll O\left(\varepsilon e^{-D_{0} / 2 \varepsilon^{2}}\right),
$$

where $D_{0}$ is the minimum value of $D \equiv\left|\mathbf{x}(s)-\mathbf{x}_{0}\right|^{2}$ for $\mathbf{x}(s) \in \partial \Omega$. The pre-exponential factor of $\varepsilon$ in A.1) is obtained by evaluating $\mathcal{I}$ in (3.48a) asymptotically using Laplace's method.

To obtain this estimate we first define an overlap region $\Omega_{p}=\{\mathbf{x} \in \Omega \mid \operatorname{dist}(\mathbf{x}, \partial \Omega)=$ $O\left(\varepsilon^{p}\right), 0<p<2$ \} between the boundary-layer region, corresponding to distances $O\left(\varepsilon^{2}\right)$ from $\partial \Omega$, and the outer region, corresponding to distances $O(1)$ from the boundary $\partial \Omega$. In $\Omega_{p}$ we use $\phi \sim \phi_{B}$ and $u_{\varepsilon} \sim u_{B}$, where $\phi_{B}$ and $u_{B}$ are defined in (2.16) and (3.8), respectively. In $\Omega \backslash \Omega_{p}$, we use $\phi \sim \varepsilon^{2} \partial_{i} u^{\infty}$ and $u_{\varepsilon} \sim u^{\infty}$, obtained from 2.10) and (1.10, respectively. We then decompose $\mathcal{K}$ into two terms as

$$
\mathcal{K}=\mathcal{K}_{1}+\mathcal{K}_{2}, \quad \mathcal{K}_{1} \equiv \int_{\Omega_{p}} \phi_{B} \mathcal{L}_{\varepsilon}\left(\partial_{i} u_{B}\right) \mathrm{d} \mathbf{x}, \quad \mathcal{K}_{2} \equiv \varepsilon^{2} \int_{\Omega \backslash \Omega_{p}} \partial_{i} u^{\infty} \mathcal{L}_{\varepsilon}\left(\partial_{i} u^{\infty}\right) \mathrm{d} \mathbf{x}
$$

We first estimate $\mathcal{K}_{1}$. The two-term boundary-layer solution $u_{B}=e^{-D /\left(4 \varepsilon^{2}\right)}\left(u_{B 0}+\varepsilon^{2} u_{B 1}+\right.$ $\left.O\left(\varepsilon^{4}\right)\right)$ is such that, formally, $\varepsilon^{2} \Delta u_{B}+u_{B} \log u_{B}=O\left(\varepsilon^{4} e^{-D /\left(4 \varepsilon^{2}\right)}\right)$. Therefore, with $u_{\varepsilon} \sim u_{B}$ in $\Omega_{p}$, we obtain

$$
\mathcal{L}_{\varepsilon}\left[\partial_{i} u_{B}\right]=\varepsilon^{2} \Delta\left(\partial_{i} u_{B}\right)+\left(1+\log u_{\varepsilon}\right) \partial_{i} u_{B}=O\left(\varepsilon^{2} e^{-D /\left(4 \varepsilon^{2}\right)}\right) .
$$

Then, using $\phi_{B} \sim \exp \left(1-D /\left(4 \varepsilon^{2}\right)\right) \Phi_{B 0}$ from 2.16, we estimate $\mathcal{K}_{1}=\int_{\Omega_{p}} e^{-D /\left(2 \varepsilon^{2}\right)} O\left(\varepsilon^{2}\right) \mathrm{d} \mathbf{x}$. Since $D \geqslant D_{0}$ and Area $\left(\Omega_{p}\right)=O\left(\varepsilon^{p}\right)$, we estimate $\mathcal{K}_{1}=e^{-D_{0} / 2 \varepsilon^{2}} O\left(\varepsilon^{2+p}\right)$. This shows that $\mathcal{K}_{1}$ satisfies the estimate $\mathcal{K}_{1} \ll O\left(\varepsilon e^{-D_{0} / 2 \varepsilon^{2}}\right)$ required in A.1 for any $p$ in $0<p<2$. Hence, we can neglect $\mathcal{K}_{1}$.

Next we estimate $\mathcal{K}_{2}$. We first write $\mathcal{L}_{\varepsilon}$ as

$$
\mathcal{L}_{\varepsilon} \phi=\varepsilon^{2} \Delta \phi+\left(1+\log u^{\infty}\right) \phi+\log \left(\frac{u_{\varepsilon}}{u^{\infty}}\right) \phi .
$$


This shows that $\mathcal{L}_{\varepsilon}\left[\partial_{i} u^{\infty}\right]=\left(\partial_{i} u^{\infty}\right) \log \left(u_{\varepsilon} / u^{\infty}\right)$, and consequently, upon using (1.10) for $u^{\infty}$, we obtain

$$
\begin{aligned}
\mathcal{K}_{2} & =\varepsilon^{2} \int_{\Omega \backslash \Omega_{p}}\left(\partial_{i} u^{\infty}\right)^{2} \log \left(\frac{u_{\varepsilon}}{u^{\infty}}\right) \mathrm{d} \mathbf{x} \\
& =c \varepsilon^{-2} \int_{\Omega \backslash \Omega_{p}}\left(x_{i}-x_{0 i}\right)^{2} e^{-\left|\mathbf{x}-\mathbf{x}_{0}\right|^{2} /\left(2 \varepsilon^{2}\right)} \log \left(\frac{u_{\varepsilon}}{u^{\infty}}\right) \mathrm{d} \mathbf{x},
\end{aligned}
$$

for some $c$ independent of $\varepsilon$. From (3.22) we obtain $u_{\varepsilon} \sim u^{\infty}$ in $\Omega \backslash \Omega_{p}$. Since $\Omega$ is convex, the boundary-layer solution $u_{B}$ is defined globally in $\Omega$, and we can estimate the ratio $u_{\varepsilon} / u^{\infty}$ as follows:

$$
\frac{u_{\varepsilon}}{u^{\infty}} \sim \frac{u_{B}}{u^{\infty}} \sim \frac{\exp \left(-D /\left(4 \varepsilon^{2}\right)+w_{0}+\varepsilon^{2} w_{1}+\cdots\right)}{\exp \left(1-\left|\mathbf{x}-\mathbf{x}_{0}\right|^{2} /\left(4 \varepsilon^{2}\right)\right)}
$$

Recalling that $w_{0}=1+\eta \chi+w_{0 p}$ and $w_{1}=-\eta^{2} / 4+w_{1 p}$, where $w_{0}$ and $w_{1}$ were defined in 3.9a and 3.11, and that $\eta=\sigma / \varepsilon^{2}$, where $\sigma$ is the distance from $\mathbf{x}$ to $\partial \Omega$, we see that A.6) becomes

$$
\frac{u_{\varepsilon}}{u^{\infty}} \sim \frac{u_{B}}{u^{\infty}} \sim \exp \left(\frac{\left|\mathbf{x}-\mathbf{x}_{0}\right|^{2}}{4 \varepsilon^{2}}-\frac{D}{4 \varepsilon^{2}}+\frac{\chi \sigma}{\varepsilon^{2}}-\frac{\sigma^{2}}{4 \varepsilon^{2}}\right) \exp \left(w_{0 p}+\varepsilon^{2} w_{1 p}+\cdots\right) .
$$

Then we use the cosine law $\left|\mathbf{x}-\mathbf{x}_{0}\right|^{2}=D+\sigma^{2}-4 \chi \sigma$ from $\left[2.7 p\right.$, together with $w_{0 p} \sim e^{-2 \chi \eta}$ for $\eta \gg 1$ as obtained from $3.9 \mathrm{~b}$. In this way, A.7p reduces to

$$
\log \left(\frac{u_{\varepsilon}}{u^{\infty}}\right) \sim \log \left(\frac{u_{B}}{u^{\infty}}\right) \sim e^{-2 \chi \sigma / \varepsilon^{2}} .
$$

Combining A.8 and A.5, and using the relation $\left|\mathbf{x}-\mathbf{x}_{0}\right|^{2}+4 \chi \sigma=D+\sigma^{2}$ from 2.7), we obtain

$$
\begin{aligned}
\mathcal{K}_{2} & =c \varepsilon^{-2} \int_{\Omega \backslash \Omega_{p}}\left(x_{i}-x_{0 i}\right)^{2} e^{-\left|\mathbf{x}-\mathbf{x}_{0}\right|^{2} /\left(2 \varepsilon^{2}\right)} e^{-2 \chi \sigma / \varepsilon^{2}} \mathrm{~d} \mathbf{x} \\
& =c \varepsilon^{-2} \int_{\Omega \backslash \Omega_{p}}\left(x_{i}-x_{0 i}\right)^{2} e^{-D /\left(2 \varepsilon^{2}\right)} e^{-\sigma^{2} /\left(2 \varepsilon^{2}\right)} \mathrm{d} \mathbf{x} .
\end{aligned}
$$

Since $D \geqslant D_{0}$ and $x_{i}-x_{0 i}$ is bounded in $\Omega \backslash \Omega_{p}$, we estimate

$$
\left|\mathcal{K}_{2}\right| \ll c_{1} \varepsilon^{-2} e^{-D_{0} /\left(2 \varepsilon^{2}\right)} \int_{\Omega \backslash \Omega_{p}} e^{-\sigma^{2} /\left(2 \varepsilon^{2}\right)} \mathrm{d} \mathbf{x},
$$

for some $c_{1}>0$ independent of $\varepsilon$. If we choose $0<p<1$ for the overlap region $\Omega_{p}$, then $\sigma=$ $\operatorname{dist}(x, \partial \Omega) \gg O(\varepsilon)$. Consequently, for this range of $p$, the integral in A.10 decays exponentially as $\varepsilon \rightarrow 0$. Therefore, for $0<p<1, \mathcal{K}_{2}$ satisfies the required estimate in A.1 and can be neglected.

\section{Appendix B. Asymptotic evaluation of an integral}

In this appendix we derive the expression for $\lambda$ in Principal Result 3.2. To do so, we define $t \geqslant 0$ by

$$
t=\frac{1}{2 \varepsilon^{2}}\left[D(s)-D_{0}\right]=\frac{D_{0}^{\prime \prime}}{4 \varepsilon^{2}}\left(s-s_{0}\right)^{2}+\frac{D_{0}^{\prime \prime \prime}}{12 \varepsilon^{2}}\left(s-s_{0}\right)^{3}+\cdots .
$$


Here we have defined $D_{0}^{(k)} \equiv D^{(k)}\left(s_{0}\right)$ for $k \geqslant 1$. We then revert the series in $(\mathrm{B} .1)$ to obtain

$$
s-s_{0}= \pm a_{1} \varepsilon t^{1 / 2}+a_{2} \varepsilon^{2} t+\cdots, \quad a_{1}=\frac{2}{\sqrt{D_{0}^{\prime \prime}}}, \quad a_{2}=-\frac{2}{3} \frac{D_{0}^{\prime \prime \prime}}{\left(D_{0}^{\prime \prime}\right)^{2}} .
$$

Here a positive (negative) sign for the leading order term is taken when $s>s_{0}\left(s<s_{0}\right)$. We then expand $\mathcal{F}_{\varepsilon}(s)$ in 3.49 in a Taylor series as $\mathcal{F}_{\varepsilon}(s)=\mathcal{F}_{00}+\mathcal{F}_{00}^{\prime}\left(s-s_{0}\right)+\mathcal{F}_{00}^{\prime \prime}\left(s-s_{0}\right)^{2} / 2+\varepsilon^{2} \mathcal{F}_{10}+$ $\cdots$. We then substitute B.2 into this series and retain terms up to $O\left(\varepsilon^{2}\right)$. This yields

$$
\mathcal{F}_{\varepsilon} \sim \mathcal{F}_{00} \pm \varepsilon a_{1} \mathcal{F}_{00}^{\prime} t^{1 / 2}+\varepsilon^{2}\left[\mathcal{F}_{10}+t\left(a_{2} \mathcal{F}_{00}^{\prime}+\frac{a_{1}^{2}}{2} \mathcal{F}_{00}^{\prime \prime}\right)\right]+\cdots
$$

Here we have labeled $\left.\mathcal{F}_{00}^{(k)} \equiv \mathcal{F}_{0}^{(k)}\right|_{s=s_{0}}$ for $k \geqslant 1$ and $\left.\mathcal{F}_{j 0} \equiv \mathcal{F}_{j}\right|_{s=s_{0}}$ for $j=0,1$. Substituting B.3 into (3.49), and by computing $\mathrm{d} s$ in (3.49) from (B.2), we find after some algebra and cancellations that

$\lambda \sim \frac{4 \varepsilon^{-4}}{\pi} e^{-D_{0} /\left(2 \varepsilon^{2}\right)}\left[\left(\varepsilon a_{1} \mathcal{F}_{00}+\varepsilon^{3} a_{1} \mathcal{F}_{10}\right) \int_{0}^{\infty} \frac{e^{-t}}{\sqrt{t}} \mathrm{~d} t+\left(3 \varepsilon^{3} a_{2} a_{1} \mathcal{F}_{00}^{\prime}+\frac{\varepsilon^{3}}{2} a_{1}^{3} \mathcal{F}_{00}^{\prime \prime}\right) \int_{0}^{\infty} \sqrt{t} e^{-t} \mathrm{~d} t\right]$.

By evaluating the integrals in (B.4, and by using (B.2) for $a_{1}$ and $a_{2}$, we obtain the estimate for $\lambda$ in 3.51).

Finally, we derive the terms in 3.52 . At $s=s_{0}$, where $D(s)$ is minimized, we have $\chi^{2}=D_{0} / 4$ and $\mathbf{e}_{s} \cdot d=0$. Hence, from 3.48b), we readily obtain 3.52a). Next, we differentiate (3.48b) for $\mathcal{F}_{0}$ to obtain

$$
\mathcal{F}_{0}^{\prime}=d_{i}^{\prime} \chi^{2}\left(\mathbf{e}_{i} \cdot \mathbf{e}_{\sigma}\right)+2 \chi \chi^{\prime} d_{i}\left(\mathbf{e}_{i} \cdot \mathbf{e}_{\sigma}\right)+d_{i} \chi^{2}\left(\mathbf{e}_{i} \cdot \partial_{s} \mathbf{e}_{\sigma}\right) .
$$

Then, by using $2 \chi^{\prime}=-\kappa \mathbf{e}_{s} \cdot \mathbf{d}=0$ and $\chi^{2}=D_{0} / 4$ at $s=s_{0}$, together with $\partial_{s} \mathbf{e}_{\sigma}=-\kappa \mathbf{e}_{s}$, we can evaluate $\mathrm{B} .5 \mathrm{~b}$ at $s=s_{0}$ to get $\left.3.52 \mathrm{~b}\right)$. To calculate $\mathcal{F}_{00}^{\prime \prime}$ we differentiate $(\mathrm{B} .5)$ with respect to $s$. After a straightforward, but lengthy calculation, we obtain the result in $3.52 \mathrm{c})$.

\section{Appendix C. Numerical solution of the metastable problem}

Here we outline the numerical method used to solve $(1.8)$ with initial data $u(\mathbf{x}, 0)=u^{\infty}\left(\mathbf{x} ; \mathbf{x}_{0}\right)$, where $u^{\infty}$ is the spike profile of $[1.10)$. The domain is taken to be the rectangle $\Omega=\{(x, y) \mid 0 \leqslant$ $\left.x \leqslant L_{x}, 0 \leqslant y \leqslant L_{y}\right\}$.

The slow motion of the spike is due to the exponentially small interaction between $u^{\infty}$ and $\partial \Omega$. Since this interaction is difficult to resolve in the $u$-variable, we consider the following problem for $w(\mathbf{x}, t)) \equiv \log [u(\mathbf{x}, t)]:$

$$
\begin{aligned}
& w_{t}=\varepsilon^{2}\left(\Delta w+w_{x}^{2}+w_{y}^{2}\right)+w, \quad \mathbf{x} \in \Omega, t>0 \\
& \partial_{n} w=0, \quad \mathbf{x} \in \partial \Omega ; \quad w(\mathbf{x}, 0)=\log \left[u^{\infty}\left(\mathbf{x} ; \mathbf{x}_{0}(0)\right)\right] .
\end{aligned}
$$

For (C.1) the initial data is not exponentially small near the boundary.

One of the best low-cost numerical methods for linear parabolic equations $u_{t}=\Delta u+f(x, y)$ is the implicit method of alternating directions. Denote the numerical grid by $\left(x_{i}, y_{j}\right)=\left(i h_{x}, j h_{y}\right)$, $i=0, \ldots, N_{x} ; j=0, \ldots, N_{y}$. The solution on the numerical grid is $u_{i j}$, and the time step is $\tau$. The 


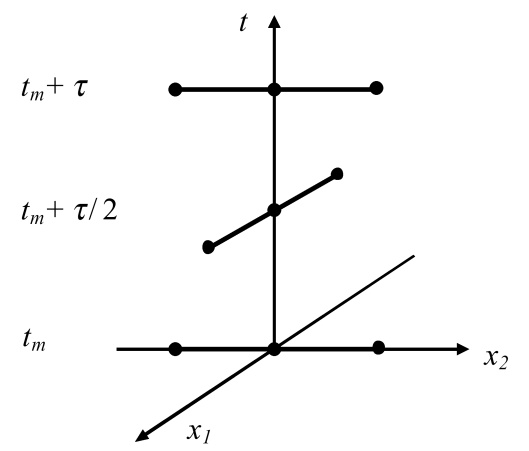

FIG. C1. The numerical pattern with $x_{1}=x$ and $x_{2}=y$.

second central difference operators in the $x$ and $y$ directions, respectively, are $\Lambda_{1} u_{i j}=h_{x}^{-2}\left(u_{i+1 j}-\right.$ $\left.2 u_{i j}+u_{i-1 j}\right)$ and $\Lambda_{2} u_{i j}=h_{y}^{-2}\left(u_{i j+1}-2 u_{i j}+u_{i j-1}\right)$.

The stages for the numerical method are shown in Fig. C1. It contains the half-step $\bar{t}=t+\tau / 2$. Given $u$ on the grid at time $t$, one first computes, on the whole grid, the intermediate value $\bar{u}$ at time $\bar{t}$, using an implicit difference in the $x$ direction and an explicit difference in the $y$ direction. The solution at the next integer time step $\hat{t}=t+\tau$, denoted by $\hat{u}_{i j}$, is implicit in the $y$ direction and explicit in the $x$ direction. In this way, we have

$$
\frac{2}{\tau}\left(\bar{u}_{i j}-u_{i j}\right)=\Lambda_{1} \bar{u}_{i j}+\Lambda_{2} u_{i j}+\bar{f}_{i j}, \quad \frac{2}{\tau}\left(\hat{u}_{i j}-\bar{u}_{i j}\right)=\Lambda_{1} \bar{u}_{i j}+\Lambda_{2} \hat{u}_{i j}+\hat{f}_{i j} .
$$

On each of the semi-steps in (C.2) a linear tridiagonal matrix system arises, which is efficiently solved with a tridiagonal solver. This fully implicit method is unconditionally stable with truncation error $O\left(\tau^{2}+h_{x}^{2}+h_{y}^{2}\right)$.

To treat the nonlinear problem (C.1) numerically, we use an explicit-implicit modification of this method. On each semi-step, the nonlinear terms are computed explicitly, while the other terms are kept fixed:

$$
\begin{aligned}
& \frac{2}{\tau}\left(\bar{w}_{i j}-w_{i j}\right)=\varepsilon^{2}\left(\Lambda_{1} \bar{w}_{i j}+\Lambda_{2} w_{i j}+\left[\frac{w_{i+1 j}-w_{i-1 j}}{2 h_{x}}\right]^{2}+\left[\frac{w_{i j+1}-w_{i j-1}}{2 h_{y}}\right]^{2}\right)+w_{i j}, \\
& \frac{2}{\tau}\left(\hat{w}_{i j}-\bar{w}_{i j}\right)=\varepsilon^{2}\left(\Lambda_{1} \hat{w}_{i j}+\Lambda_{2} \bar{w}_{i j}+\left[\frac{\bar{w}_{i+1 j}-\bar{w}_{i-1 j}}{2 h_{x}}\right]^{2}+\left[\frac{\bar{w}_{i j+1}-\bar{w}_{i j-1}}{2 h_{y}}\right]^{2}\right)+\bar{w}_{i j} .
\end{aligned}
$$

The condition $\partial_{n} w=0$ on the rectangle boundary is approximated by introducing auxiliary additional sets of grid points with $i=-1, j=-1, i=N_{x}+1$ and $j=N_{y}+1$ respectively, and by setting the solution values at these auxiliary grid points to be equal to the solution values at points symmetric with respect to the boundary.

\section{Acknowledgements}

A.F.C. thanks NSERC (Canada) and the Killam foundation for postdoctoral fellowship support. M.J.W. thanks NSERC for the grant support and Prof. Juncheng Wei of the Chinese University of Hong Kong for some early discussions relating to this problem. 


\section{REFERENCES}

1. Berestycki, H. Kagan, L., Kamin, S., \& Sivashinsky, G. Metastable behavior of premixed gas flames in rectangular channels. Interfaces Free Bound. 6 (2004), 423-438. Zbl pre02148306 MR 2111564

2. Berestycki, H. KAMIN, S., \& SiVAShinsky, G. Nonlinear dynamics and metastability in a Burgerstype equation. C. R. Acad. Sci. Paris 321 (1995), 185-190. Zbl 0838.35112 MR 1345444

3. Berestycki, H. KAmin, S., \& Sivashinsky, G. Metastability in a flame front evolution equation. Interfaces Free Bound. 3 (2001), 361-392. Zbl 0991.35097| MR 1869585

4. Chen, X. \& Kowalczy , M. Slow dynamics of interior spikes in the shadow Gierer-Meinhardt system. Adv. Differential Equations 6 (2001), 847-872. Zbl 1032.35025 MR 1828999

5. Del Pino, M. \& Felmer, P. Spike-layered solutions of singularly perturbed elliptic problems in a degenerate setting. Indiana Univ. Math. J. 48 (1999), 883-898. Zbl 0932.35080 MR 1736974

6. IRON, D. \& WARD, M. J. A metastable spike solution for a non-local reaction-diffusion model. SIAM J. Appl. Math. 60 (2000), 778-802. Zbl 0956.35011 MR 1740850

7. IRON, D. \& WARD, M. J. The dynamics of boundary spikes for a non-local reaction-diffusion model. Eur. J. Appl. Math. 11 (2000), 491-514. Zbl 0971.92002 MR 1799923

8. Kamin, S. Berestycki, H., Kagan, L., \& Sivashinsky, G. Metastable behavior of premixed gas flames. Elliptic and Parabolic Problems, Progr. Nonlinear Differential Equations Appl. 63, Birkhäuser, Basel (2005), 319-328. Zbl 1083.35048 MR 2176724

9. Keller, J. B. \& Lewis, R. M. Asymptotic methods for partial differential equations: the reduced wave equation and Maxwell's equations. Surveys in Applied Mathematics, Vol. 1, J. B. Keller et al. (eds.), Plenum Press, New York (1995), 1-82. Zbl 0848.35068 MR 1366207

10. Mikishev, A. B. \& Sivashinsky, G. I. Quasi-equilibrium in upward propagating flames. Phys. Lett. A 175 (1993), 409-414. MR 1215405

11. Morse, P. M. \& FeshBACH, P. Methods of Theoretical Physics. Vol. 2, McGraw-Hill, New York (1953). Zbl 0051.40603 MR 0059774

12. NI, W. M. Qualitative properties of solutions to elliptic problems. Stationary Partial Differential Equations, Vol. 1, Handbook of Differential Equations, North-Holland, Amsterdam (2004), 157-233. Zbl pre02163592 MR 2103689

13. NI, W. M. \& TAKAGI, I. Locating the peaks of least energy solutions to a semilinear Neumann problem. Duke Math J. 70 (1993), 247-281. Zbl 0796.35056 MR 1219814

14. OU, C. H. \& WARD, M. J. A metastable flame-front interface and Carrier's problem. J. Comput. Appl. Math. 190 (2006), 354-375. Zbl 05015186 MR 2209513

15. RAKIB, Z. \& SiVASHINSKy, G. I. Instabilities in upward propagating flames. Combust. Sci. Tech. 54 (1987), 69-84.

16. SUn, X. \& WARD, M. J. Metastability for a generalized Burgers equation with applications to propagating flame-fronts. Eur. J. Appl. Math. 10 (1999), 27-53. Zbl 0919.35122 MR 1685819

17. WARD, M. J. Metastable bubble solutions for the Allen-Cahn equation with mass conservation. SIAM J. Appl. Math. 56 (1996), 1247-1279. Zbl 0870.35011 MR 1409118

18. WARD, M. J. An asymptotic analysis of localized solutions for some reaction-diffusion models in multidimensional domains. Stud. Appl. Math. 97 (1996), 103-126. Zbl 0932.35059 MR 1395845

19. WARD, M. J. Metastable dynamics and exponential asymptotics in multi-dimensional domains. Multiple Time-Scale Dynamical Systems, IMA Vol. Math. Appl. 122, C. K. R. T. Jones and A. Khibnik (eds.) (2000), 233-260. Zbl 1007.35002 MR 1846579

20. WARD, M. J. Spikes for singularly perturbed reaction-diffusion systems and Carrier's problem. Differential Equations and Asymptotic Theory in Mathematical Physics, H. Chen and R. Wong (eds.), Ser. Anal. 2, World Sci., Singapore (2005), 100-188. Zbl 05192179 MR 2161972 
21. WEI, J. On single interior spike solutions for the Gierer-Meinhardt system: Uniqueness and stability estimates. Eur. J. Appl. Math. 10 (1999), 353-378. Zbl 1014.35005 MR 1713076

22. WEI, J. On the boundary spike layer solutions of singularly perturbed semilinear Neumann problem. J. Differential Equations 134 (1997), 104-133. Zbl 0873.35007 MR 1429093

23. WEI, J. On the interior spike layer solutions to a singularly perturbed Neumann problem. Tohoku Math. J. 50 (1998), 159-178. Zbl 0918.35024 MR 1622042

24. Wong, R. Asymptotic Approximations of Integrals. Academic Press, San Diego, CA (1989). Zbl 0679.41001 MR 1016818 